TRANSACTIONS OF THE

AMERICAN MATHEMATICAL SOCIETY

Volume 352, Number 11, Pages 5317-5355

S 0002-9947(00)02453-3

Article electronically published on May 12, 2000

\title{
SEMI-CLASSICAL LIMIT FOR RANDOM WALKS
}

\author{
URSULA POROD AND STEVE ZELDITCH
}

\begin{abstract}
Let $(G, \mu)$ be a discrete symmetric random walk on a compact Lie group $G$ with step distribution $\mu$ and let $T_{\mu}$ be the associated transition operator on $L^{2}(G)$. The irreducibles $V_{\rho}$ of the left regular representation of $G$ on $L^{2}(G)$ are finite dimensional invariant subspaces for $T_{\mu}$ and the spectrum of $T_{\mu}$ is the union of the sub-spectra $\sigma\left(T_{\mu} 1_{V_{\rho}}\right)$ on the irreducibles, which consist of real eigenvalues $\left\{\lambda_{\rho 1}, \ldots, \lambda_{\rho \operatorname{dim} V_{\rho}}\right\}$. Our main result is an asymptotic expansion for the spectral measures

$$
m_{\rho}^{\mu}(\lambda):=\frac{1}{\operatorname{dim} V_{\rho}} \sum_{j=1}^{\operatorname{dim} V_{\rho}} \delta\left(\lambda-\lambda_{\rho j}\right)
$$

along rays of representations in a positive Weyl chamber $\mathbf{t}_{+}^{*}$, i.e. for sequences of representations $k \rho, k \in \mathbb{N}$ with $k \rightarrow \infty$. As a corollary we obtain some estimates on the spectral radius of the random walk. We also analyse the fine structure of the spectrum for certain random walks on $U(n)$ (for which $T_{\mu}$ is essentially a direct sum of Harper operators).
\end{abstract}

\section{INTRODUCTION}

The spectral theory of a random walk $(G, \mu)$ on a compact Lie group $G$ is concerned with the spectrum of the Markov (or transition) operator

$$
\begin{gathered}
T_{\mu}: L^{2}\left(G, d \mu_{0}\right) \rightarrow L^{2}\left(G, d \mu_{0}\right), \\
T_{\mu}(f)=\mu \star f
\end{gathered}
$$

where $d \mu_{0}$ is a Haar measure on $G$ and where $\mu$ is a positive measure of mass 1 . If $\mu$ is symmetric, i.e., $d \mu(g)=d \mu\left(g^{-1}\right)$ for all $g \in G$, the operator $T_{\mu}$ is self-adjoint on $L^{2}\left(G, d \mu_{0}\right)$. Moreover, if

$$
L^{2}\left(G, d \mu_{0}\right)=\bigoplus_{\rho \in \hat{G}} V_{\rho} \otimes V_{\rho}^{*} \quad(\hat{G}=\text { unitary dual })
$$

is the decomposition into irreducibles of the left regular representation of $G$ on $L^{2}\left(G, d \mu_{0}\right)$, then

$$
T_{\mu}: V_{\rho} \rightarrow V_{\rho} \quad \text { for all } \rho \in \hat{G},
$$

Received by the editors December 12, 1997 and, in revised form, August 25, 1998.

1991 Mathematics Subject Classification. Primary 60B15, 60J15, 22E30; Secondary 58F06.

Supported by the Miller Institute for Basic Research in Science and partially by NSF grant \#DMS-9404637. 
that is, all irreducibles are invariant subspaces for $T_{\mu}$. It follows that $\sigma\left(T_{\mu}\right)$, the spectrum of $T_{\mu}$, has the form

$$
\sigma\left(T_{\mu}\right)=\bigcup_{\rho \in \hat{G}} \sigma\left(T_{\mu} 1_{V_{\rho}}\right)
$$

where $T_{\mu} 1_{V_{\rho}}$ is the restriction of $T_{\mu}$ to $V_{\rho}$. Equivalently, we have

$$
\sigma\left(T_{\mu}\right)=\bigcup_{\rho \in \hat{G}} \sigma(\hat{\mu}(\rho))
$$

where

$$
\hat{\mu}(\rho)=\int_{G} \rho(g) d \mu(g)
$$

is the Fourier transform of $\mu$ at $\rho$. The decomposition (0.2) shows that $\sigma\left(T_{\mu}\right) \subset$ $[-1,1]$ consists entirely of eigenvalues. The main purpose of this paper is to exploit it further to determine some fine structure properties of the spectrum.

To state the problem more precisely, we first recall that a unitary irreducible representation $\rho$ of $G$ is uniquely determined by its highest weight, which for convenience we will also denote by $\rho$. Hence the unitary dual is parametrized

$$
\hat{G}=I^{*} \cap \mathbf{t}_{+}^{*}
$$

by the intergral points $I^{*}$ in a positive Weyl chamber of the dual of a Cartan subalgebra t. For each $\rho \in I^{*} \cap \mathbf{t}_{+}^{*}$ we associate to $\sigma(\hat{\mu}(\rho))=\left\{\lambda_{\rho i}: i=1,2, \ldots, \operatorname{dim} V_{\rho}\right\}$ the probability measure

$$
m_{\rho}^{\mu}(\lambda):=\frac{1}{\operatorname{dim} V_{\rho}} \sum_{j=1}^{\operatorname{dim} V_{\rho}} \delta\left(\lambda-\lambda_{\rho j}\right)
$$

in $\mathbb{R}$. Our interest is in determining the asymptotic properties of the measures $m_{\rho}^{\mu}$ as $|\rho| \rightarrow \infty$ along rays in $\hat{G}$, i.e., for sequences of representations $k \rho, k \in \mathbb{N}$ with $k \rightarrow \infty$. This generalizes the analysis of asymptotics of Fourier coefficients of measures on the circle group $S^{1}$. Since the limit along rays has many (well-known) analogies to the semi-classical limit in quantum mechanics, we view the problem as that of determining the semi-classical limit of the random walk.

Asymptotic expansions for $m_{k \rho}^{\mu}$ cannot be expected, and indeed do not exist, for an arbitrary measure $\mu$ on $G$. For instance, in the case of $S^{1}$ there are just two rays in $\hat{S^{1}} \simeq \mathbb{Z}, \mathbb{Z}^{+}$and $\mathbb{Z}^{-}$, and $m_{ \pm k}^{\mu}(\lambda)=\delta(\lambda-\hat{\mu}( \pm k))$. Under the symmetry condition, $\hat{\mu}(k)=\hat{\mu}(-k)$, so we have

$$
\mu=1+2 \sum_{k \geq 1} \hat{\mu}(k) \cos k \theta
$$

and any asymptotic expansion

$$
\hat{\mu}(k) \sim a_{0} k^{m}+a_{-1} k^{m-1}+\ldots \quad(k \rightarrow \infty)
$$

of the Fourier coefficients $\hat{\mu}(k)$ would immediately entail an asymptotic expansion (in the sense of singularities)

$$
\mu \sim 1+a_{0} \Re \chi_{m}+a_{-1} \Re \chi_{m-1}+\ldots
$$


of $\mu$ in terms of the homogeneous distributions

$$
\chi_{r}(\theta)=\sum_{k=0}^{\infty} k^{r} e^{i k \theta}
$$

on $S^{1}\left(\Re \chi_{r}\right.$ denotes the real part of $\left.\chi_{r}\right)$. Such $\mu$ are clearly of a restricted class. However, this class is a very natural one which generalizes to any group - it is the class of "Lagrangean" measures of modern Fourier analysis (aka the theory of Fourier integral operators; see [Ho, IIV] [D]). For such measures, asymptotic expansions similar to the one above can be expected.

Many random walks $(G, \mu)$ of interest in probability theory fit into this framework, for example, discrete random walks (i.e., with supp $\mu \subset G$ discrete) or random walks with $\mu$ supported on a conjugacy class (as studied in $[\mathrm{R}],[\mathrm{P}]$ ). For such random walks it is possible to apply the methods of microlocal analysis to study the spectrum and smoothing properties of $T_{\mu}$. In this paper we concentrate on discrete random walks, i.e. where $\mu$ is a discrete measure

$$
\mu=\frac{1}{m_{\mu}} \sum_{i=1}^{n} c_{i}\left(\delta_{a_{i}}+\delta_{a_{i}^{-1}}\right)
$$

with $m_{\mu}=2 \sum_{i=1}^{n} c_{i}$. In the sequel [PZ] we extend the methods and results to certain continuous Lagrangean random walks, such as $\delta$-functions on positively curved hypersurfaces or on sums of conjugacy classes. Discrete random walks are much simpler technically than continuous random walks and hopefully provide a clean introduction to the microlocalization of random walks to rays of representations.

Let us explain this notion in more detail before describing our methods and results. The key idea is to simplify the study of a random walk on a compact Lie group by localizing it to a ray in the cotangent bundle $T^{*} G$ or in the unitary dual $\hat{G}$. The motivation for such a localization is that the Fourier transform $\hat{\mu}(\rho)$ can behave differently in different directions as $\rho \rightarrow \infty$ and one would like to isolate the behaviour in each direction. Each ray correponds to a different co-adjoint orbit, and the localization to a ray restricts the dynamics of the random walk to this orbit. This kind of behaviour is well-known in the Fourier analysis of surfacecarrying measures on $\mathbb{R}^{n}$ (see e.g. [Stein, VIII: §5]) and arises for essentially the same reasons on any Lie group. It does not seem to have been studied before in the setting of non-abelian Lie groups (see however the paper of Taylor-Uribe [TU] for a microlocal analysis of certain central measures).

Thus, our goal is to microlocalize a discrete random walk $T_{\mu}$ to a ray of reprsentations $\mathbb{N} \rho$ and to determine the asymptotic behaviour of its Fourier transform as encoded by the spectral measures $m_{r \rho}^{\mu}$. Since this only involves the $L^{2}$ theory of $T_{\mu}$, we are free to conjugate to a realization of the ray $\mathbb{N} \rho$ best adapated to the problem of determining the semi-classical limit of the spectral measures $m_{r \rho}^{\mu}$. The relevant construction is the homogeneous generalization of the Kirillov-Kostant orbit theory due to Guillemin and Sternberg GS1].

Recall that in the classical Borel-Weil-Bott-Kirillov-Kostant theory, an irreducible representation $\left(\rho, V_{\rho}\right)$ corresponds to the classical "phase space" $\mathcal{O}_{\rho}$, the coadjoint orbit of $\rho$ in $g^{*}$. In the homogeneous quantization theory of Guillemin and Sternberg [GS1], the entire ray $\mathbb{N} \rho$ corresponds to the Hardy subspace $H^{2}\left(B_{\rho}\right) \subset$ $L^{2}\left(B_{\rho}\right)$ where $B_{\rho}$ is the so-called "prequantum" $S^{1}$-bundle over $\mathcal{O}_{\rho}$. We defer the precise definitions of these objects to $\S 2$. For the time being, the main point is 
that $B_{\rho}$ is a homogeneous space of $G$, and that $H^{2}\left(B_{\rho}\right) \equiv \bigoplus_{r=0}^{\infty} V_{r \rho}$. Thus, we can determine the semi-classical asymptotics of $m_{r \rho}^{\mu}$ along the ray by studying the concrete random walk $\left(G, \mu, H^{2}\left(B_{\rho}\right)\right)$. The spectrum of $T_{\mu}$ on any homogeneous space, in particular on $G$, can then be synthesized from these special, but fundamental, cases. Concrete examples of such rays of representations will be presented in $\S 4$, along with rays such as $L^{2}\left(S^{n}\right)$ for $G=S O(n+1)$ which do not arise from this construction.

Let us now state our results. In the following, we identify irreducibles with their highest weights $\rho$, which are lattice points in a positive Weyl chamber $\mathbf{t}_{+}^{*}$. The notation interior $\left(\mathbf{t}_{+}^{*}\right)$ stands for the interior of the Weyl chamber. There is a slight distinction in the results between interior weights $\rho$ and weights lying on the walls of the Weyl chamber (which we will refer to as 'wall weights.)' For the sake of brevity, we concentrate on the interior case and do not describe the possible behaviours of wall weights. However, we should point out that the results for wall weights are often identical to the results for interior weights. For instance, in the case of $G=U(n)$ only scalar weights behave differently from interior weights (see the remark in $\S 3)$.

Main Theorem. Let $\mu=\frac{1}{m_{\mu}} \sum_{i=1}^{n} c_{i}\left(\delta_{a_{i}}+\delta_{a_{i}^{-1}}\right)$ be a discrete probability measure on a compact Lie group $G$. Then the measures $m_{r \rho}^{\mu}$ possess asymptotic expansions as $r \rightarrow \infty$ in the following sense: there exist measures $d m^{\mu, z}$ on $\mathbb{R}$ (parametrized by $z \in Z_{G}$ ) so that for any polynomial $f$ on $\mathbb{R}$,

(a) Interior case: If $\rho \in$ interior $\left(\mathbf{t}_{+}^{*}\right)$, then

$$
\int_{\mathbb{R}} f d m_{r \rho}^{\mu}=\sum_{z \in Z_{G} \cap \Gamma} \chi_{\rho}(z)^{r} \int_{\mathbb{R}} f d m^{\mu, z}+O\left(r^{-1}\right)
$$

where

(i) $Z_{G}$ denotes the center of $G$;

(ii) $\Gamma=\left\langle a_{i}\right\rangle \subset G$ denotes the subgroup generated by $\left\{a_{i}\right\}$;

(iii) $\chi_{\rho}$ denotes the highest weight character of $\rho$;

(iv) $(e) d m^{\mu, e}$ is the positive measure on $[-1,1]$ determined by the moments

$$
\int_{\mathbb{R}} x^{p} d m^{\mu, e}=\mu^{* p}(e)
$$

(iv) $(z) d m^{\mu, z}$ is the measure on $[-1,1]$ with the moments

$$
\int_{\mathbb{R}} x^{p} d m^{\mu, z}=\mu^{* p}(\{z\}) ;
$$

(v) For a polynomial $f$ the sum on the right side of (0.5) is always finite. In the case where $Z_{G} \cap \Gamma$ is a finite set, then for any $f \in C(\mathbb{R})$ one has

$$
\int_{\mathbb{R}} f d m_{r \rho}^{\mu}=\sum_{z \in Z_{G} \cap \Gamma} \chi_{\rho}(z)^{r} \int_{\mathbb{R}} f d m^{\mu, z}+o(1) .
$$

(b) Wall case: Suppose $\rho$ is a wall weight, and let

(vi) $Z_{\rho}:=\bigcap_{g \in G} g^{-1} G_{\rho} g$, where $G_{\rho}$ is the stabilizer group of $\rho$. Then analogously to the interior case one has for any polynomial $f$ that 


$$
\int_{\mathbb{R}} f d m_{r \rho}^{\mu}=\sum_{z \in Z_{\rho} \cap \Gamma} \chi_{\rho}(z)^{r} \int_{\mathbb{R}} f d m^{\mu, z}+o(1)
$$

with (ii)-(v) unchanged.

We observe that the measures $d m_{r \rho}^{\mu}$ do not generally tend to a weak limit as $r \rightarrow \infty$ due to oscillations in the asymptote on the right side of (0.5). One may obtain weak limits along subsquences $r_{k} \rho$ with fixed values of $\chi_{\rho}^{r_{k}}$ (cf. Corollary 2).

The main assertion is (0.5) and the other assertions in (i)-(v) are simple consequences of it and of general facts about convolution powers. The finiteness of the sum on the right side of $(0.5)$ for polynomial $f$ follows immediately from the finiteness of the support of $\mu$. Moreover, it will follow from the proof that the asymptote is just the first term of a complete asymptotic expansion.

For a general $f \in C(\mathbb{R})$ the sum on the right may be infinite and may be hard to control. The fact that $m^{\mu, z}$ is a measure of finite mass for $z \neq e$ will be proved in remark (3) below. The main point is that the convolution algebra generated by $\left\{\mu, \delta_{e}\right\}$ is a commutative $\mathrm{C}^{*}$ algebra with unit and hence may be identified with the space continuous functions on the compact Hausdorff space $\sigma\left(T_{\mu}\right)$. The measure $m^{\mu, z}$ may be identified with a continuous linear functional on the spectrum. Once this is established the asymptotics in (0.5) lead immediately to the asymptotic in (v) by approximating continuous functions by polynomials.

Let us now derive a number of consequences from the Main Theorem.

First, consider the asymptote $\sum_{z \in Z_{G} \cap \Gamma} \chi_{\rho}(z)^{r} d m^{\mu, z}$ in the interior case. It is natural to lump together the terms with a fixed value of $\chi_{\rho}(z)$. We therefore set

$$
\Theta_{\rho}:=\left\{\theta \in[0,2 \pi): \exists z \in Z_{G} \cap \Gamma, \chi_{\rho}(z)=e^{i \theta}\right\}
$$

which is evidently a countable subset of angles $\left\{\theta_{j}\right\}$. For each $\theta \in \Theta_{\rho}$ we also put

$$
Z_{\rho, \theta}:=\left\{z \in Z_{G} \cap \Gamma: \chi_{\rho}(z)=e^{i \theta}\right\} .
$$

Then the asymptote can be re-written in the form:

$$
\sum_{z \in Z_{G} \cap \Gamma} \chi_{\rho}(z)^{r} d m^{\mu, z}=\sum_{\theta_{j} \in \Theta_{\rho}} e^{i r \theta_{j}} d m_{\theta_{j}}^{\mu}
$$

with

$$
d m_{\theta_{j}}^{\mu}=\sum_{z \in Z_{\rho, \theta_{j}}} d m^{\mu, z} .
$$

As is typical in the world of trace formulae, the terms in (0.9.2) can possibly cancel each other out. This certainly does not happen in the case of $\theta=0$ since we have

$$
d m_{o}^{\mu}=\lim _{N \rightarrow \infty} \frac{1}{N} \sum_{n=0}^{N} d m_{n \rho}^{\mu},
$$

i.e. the limit spectral measure with $\theta=0$ is the Cesaro mean of the spectral measures along the ray. Since it is an average of positive measures it must be positive. However, the other limit spectral measures are given by

$$
d m_{\theta_{j}}^{\mu}=\lim _{N \rightarrow \infty} \frac{1}{N} \sum_{n=0}^{N} e^{-i n \theta_{j}} d m_{n \rho}^{\mu}
$$


and are consequently signed averages. There is no obvious reason why they cannot cancel out. To take this possibility into account, we henceforth write

$$
\Theta_{\rho}^{*}:=\left\{\theta \in \Theta_{\rho}: d m_{\theta}^{\mu} \neq 0\right\} .
$$

It is evident from the Main Theorem and from $(0.10 . \mathrm{j})$ that the angles $\theta_{j} \in$ $\Theta_{\rho}^{*}$ and the measures $d m_{\theta_{j}}^{\mu}$ are spectral invariants of the random walk $T_{\mu}$ along the ladder $\mathbb{N} \rho$. They are analogous to the so-called principal band invariants of a self-adjoint Toeplitz operator on contact manifold with periodic characteristic flow, or of the Laplacian on a Zoll manifold (cf. BdMG, §13] for background and references). However, the Markov operator $T_{\mu}$ is a kind of Fourier Integral Toeplitz operator associated to a Markov chain (the random walk), whereas the previously studied Toeplitz or pseudodifferential operators have no dynamical aspect (they are associated to the identity map). Hence the analogy only goes so far. In particular, we have not one band invariant, but a whole family $d m_{\theta_{j}}^{\mu}$ of band invariants. To give them a name, we will refer to them as the limit spectral measures of the random walk on the ray. The name is justified by the following:

Corollary 1. In the notation of the Main Theorem, suppose that $\rho$ is an interior weight. Then

$$
\bigcup_{\theta_{j} \in \Theta_{\rho}} \operatorname{supp}\left(d m_{\theta_{j}}^{\mu}\right) \subset \overline{\sigma\left(T_{\mu} 1_{\mathbb{N} \rho}\right)}
$$

$\bar{S}$ denotes the closure of the set $S$ ).

The proof is just to observe that if $\lambda \notin \overline{\sigma\left(T_{\mu} 1_{\mathbb{N} \rho}\right)}$, then there is an open set $(\lambda-\epsilon, \lambda+\epsilon)$ disjoint from $\overline{\sigma\left(T_{\mu} 1_{\mathbb{N} \rho}\right)}$ and hence all the band spectral measures $d m_{r \rho}^{\mu}$ annihilate the functions in $C_{c}(\lambda-\epsilon, \lambda+\epsilon)$. Hence so must each limit measure $d m_{\theta_{j}}^{\mu}$. The result in the wall case is analogous and is left to the reader to formulate.

We note that the converse $\subset$ relation is a very difficult problem, since a priori the limit spectral measures only capture points of density in the spectrum of the random walk. Whether or not all points of $\sigma\left(T_{\mu} 1_{\mathbb{N} \rho}\right)$ are points of density is a question that requires a detailed study in each special case.

Two extreme cases occur when $\chi_{\rho}(z) \equiv 1$ and when $\chi_{\rho}(z) \neq 1$ for $z \neq e$. In the first case we obviously have

Corollary 2. If $\chi_{\rho}(z)=1$ for all $z \in Z_{G} \cap$ supp $\mu_{p}$ (for example, if $Z_{G}$ is trivial), then, for interior $\rho$, there exists a weak limit

$$
m_{r \rho}^{\mu} \stackrel{w^{*}}{\longrightarrow} m^{\mu}
$$

with moments

$$
\int_{\mathbb{R}} x^{p} d m^{\mu}=\sum_{z \in Z_{G}} \mu^{* p}(\{z\}) .
$$

In the second case, we can deduce results about the spectral radius

$$
\beta_{\rho}^{*}:=\sup \left\{|\lambda|: \lambda \in \sigma\left(T_{\mu} 1_{\mathbb{N} \rho}\right)\right\}
$$

of the random walk $(G, \mu)$ along the ray $\mathbb{N} \rho$. The key point here is that the limit measures $d m^{z, \mu}$ depend only on the discrete group $\Gamma:=\left\langle a_{i}^{ \pm 1}, i=1, \ldots, n\right\rangle$, on the generating set $A=\left\{a_{i}\right\}$ and on the set $Z_{G}$. In particular, the limit measure $d m^{\mu, e}$ corresponding to the identity element $e$ is the Kesten spectral measure of the random walk $(\Gamma, \mu)$ defined by $\mu$ on $l^{2}(\Gamma)$. As is well-known, this spectral measure 
encodes many properties of the pair $(\Gamma, A)$. See $[\mathrm{KV}]$ and $[\mathrm{HRV}$ for background. From the known results on the spectrum of $(\Gamma, \mu)$, we conclude:

Corollary 3. Suppose $\chi_{\rho}(z) \neq 1$ for $z \in Z_{G} \cap \Gamma \backslash\{i d\}$. Then, with the above notation and assumptions:

(i) Suppose $\Gamma$ is an amenable group. Then $\beta_{\rho}^{*}=1$.

(ii) Suppose $\Gamma$ is a free group and $\left\{a_{i}, i=1, \ldots, n\right\}$ is a free set of generators. Then $\left[-\frac{\sqrt{2 n-1}}{n}, \frac{\sqrt{2 n-1}}{n}\right] \subseteq \overline{\sigma\left(T_{\mu} 1_{\mathbb{N} \rho}\right)}$.

Remarks. (1) The Main Theorem may be viewed as connecting the spectral theory of the random walk determined by $\mu$ along rays of representations of $G$ with the spectral theory of the random walk $(\Gamma, \mu)$ defined by $\mu$ on $l^{2}(\Gamma)$. Essentially, we are looking at different representations of the element $T_{\mu}=\frac{1}{m_{\mu}} \sum c_{i} a_{i} \in \mathbb{C}[\Gamma]$. For the random walk $(\Gamma, \mu)$, we are considering the left regular representation $\lambda$ of $\Gamma$ on $l^{2}(\Gamma)$. Along the ray, we are considering the representation $H^{2}\left(B_{\rho}\right)$ of $G$, restricted to $\Gamma$. For simplicity of notation, let us denote this restricted representation of $\Gamma$ by $\pi_{\rho}$. Then in favorable cases, our results allow us to compare the spectra $S p \lambda\left(T_{\mu}\right)$ and $S p \pi_{\rho}\left(T_{\mu}\right)$.

(2) To enlarge on the comparison, we consider it from the $C^{*}$-algebraic point of view (cf. [HRV]). Given a unitary representation $\pi$ of $\Gamma$ on a Hilbert space $\mathcal{H}$ we can take the norm closure of $\mathbb{C}[\Gamma]$ in the algebra $\mathcal{L}(\mathcal{H})$ of bounded operators on $\mathcal{H}$, to obtain a $C^{*}$ algebra $C_{\pi}^{*}(\Gamma)$. In the case of the left regular representation, we get the reduced $C^{*}$-algebra $C_{\lambda}^{*}(\Gamma)$. In the case of $\pi_{\rho}$ we get a representation $C_{\pi_{\rho}}^{*}$.

Consider for instance the spectral measure $d m^{\mu, e}$ from the $C^{*}$ point of view. Its moments may be expressed in the form

$$
\int_{\mathbb{R}} x^{p} d m^{\mu, e}=\left\langle\lambda\left(T_{\mu}\right)^{p} \delta_{e}, \delta_{e}\right\rangle .
$$

The linear functional $\tau(A)=\left\langle\lambda(A) \delta_{e}, \delta_{e}\right\rangle$ on $\mathbb{C}[\Gamma]$ is continuous with respect to the operator norm of $\lambda(A)$ on $l^{2}(\Gamma)$ and hence extends to a continuous trace on $C_{\lambda}^{*}(\Gamma)$. It further extends to a continuous trace on the weak closure $W_{\lambda}^{*}(\Gamma)$. In terms of the spectral resolution $d E_{x}$ of $\lambda\left(T_{\mu}\right)$ we may write

$$
m^{\mu, e}(B)=\tau\left(E_{B}\right)
$$

for any Borel $B \subset \mathbb{R}$. It is known that the support of this measure $m^{\mu, e}$ equals the spectrum $S p_{\lambda}\left(T_{\mu}\right)$ of $\lambda\left(T_{\mu}\right)$ as an element of $C_{\lambda}^{*}(\Gamma)$ (cf. [HRV, Proposition 5]). This fact allows one to relate $S p_{\lambda}\left(T_{\mu}\right)$ to the properties of $(\Gamma, A)$ (loc. cit.)

The fact that $m^{\mu, e}$ arises in the asymptotics of $m_{r \rho}^{\mu}$ also allows us to relate $S p_{\pi_{\rho}}\left(T_{\mu}\right)$ to the properties of $\left(\Gamma, A, \pi_{\rho}\right)$. Corollary 3 above gives one example. The other spectral measures arising in the asymptotics can be used in a similar way, at least when the oscillatory factors $\chi_{\rho}(z)^{r}$ can be separated out from each other.

(3) We note that the other spectral measures $m^{\mu, z}$ with $z \neq e$ also arise from traces on $C_{\lambda}^{*}(\Gamma)$. They are defined by $\tau^{z}(a):=\left\langle a \delta_{e}, \delta_{z}\right\rangle$. In other words, $\tau^{z}(\lambda(\gamma))=$ 1 if $\gamma z=e$ and is otherwise zero.

The trace condition $\tau^{z}(a b)=\tau^{z}(b a)$ follows easily from the condition $z \in Z_{G}$, and continuity on $C_{\lambda}^{*}(\Gamma)$ is obvious. However, $\tau^{z}(I)=0$ if $z \neq e$ so the traces are not states on the algebra. 
The traces $\tau^{z}$ induce measures on $S p \lambda\left(T_{\mu}\right)$ as in the case of $z=e$; namely, we define $m^{\mu, z}$ on $C\left(S p \lambda\left(T_{\mu}\right)\right)$ by

$$
\int f d m^{\mu, z}:=\tau^{z}\left(f\left(\lambda\left(T_{\mu}\right)\right)\right)
$$

Since $\| f\left(\lambda\left(T_{\mu}\right)\|=\| f \|_{\infty}\right.$ (the sup-norm on the set $\operatorname{Spec}\left(\lambda\left(T_{\mu}\right)\right)$ ), it is evident that $m^{\mu, z}$ does actually define a measure on $\operatorname{Sp} \lambda\left(T_{\mu}\right)$.

(4) The traces $\tau^{z}$ originally arise as residue (Dixmier) traces on the algebra of Toeplitz operators on $H^{2}\left(B_{\rho}\right)$. They are in general not continuous traces on the $C^{*}$ algebra (norm closure) of the Toeplitz algebra. When restricted to $C_{\pi_{\rho}}^{*}$ they may be viewed as defining unbounded traces on this algebra.

Examples. Examples of rays of representations of compact semi-simple Lie groups and of their discrete random walks will be given in $\S 4$. In particular, we will analyze in detail the spectrum of a specific discrete random walk on the unitary group (the pseudo-Harper operator) by means of the spectral measures obtained in the Main Theorem.

For the time being, let us consider some well-known examples from the literature on the spectral theory of discrete random walks on Lie groups.

Ex. 1. A special case of a discrete random walk on a ray of representations is given by the Hecke operators on $L^{2}\left(S^{2}\right)$ studied by Lubotzky, Phillips, and Sarnak [LPS] (see also [CV]) In this example, the group is $G=S O(3)$ and $L^{2}\left(S^{2}\right)$ consists of just one ray. The random walk has transition operator $T=\frac{1}{6} \sum T_{a_{i}}+T_{a_{i}^{-1}}$ where the $a_{i}$ are rotations around three perpendicular axes and with angle $\alpha$ given by $\cos \alpha=-\frac{3}{5}$. The group $\Gamma$ is a free group, and the rate of equidistribution of orbits of the random walk is optimal. Since $G$ has trivial center, only the measure corresponding to $z=e$ shows up in limit spectral measure, i.e. the limit spectral measure of this random walk is the Kesten spectral measure for the left regular representation.

Our methods could also be extended to non-compact Lie groups such as $G=$ $S L(2, \mathbb{R})$, and would then apply to the (true) Hecke operators $T_{p}$, acting in the discrete or continuous ray (series) of representations of the quotient $G / S L(2, \mathbb{Z})$. The results are just as in the previous example.

Ex. 2. A well-known example of a discrete random walk on the three-dimensional reduced Heisenberg group $N_{\mathbb{R}}$ is given by the measure $\frac{1}{4}\left[\delta_{U}+\delta_{U}^{*}+\delta_{V}+\delta_{V}^{*}\right]$, where $U, V$ are elements satisfying $U V=e^{i \theta} V U$. The corresponding transition operator is the Harper operator $H_{\theta}=\frac{1}{4}\left[U+U^{*}+V+V^{*}\right]$. It is much-studied as a model for a periodic Schrodinger operator in a magnetic field; see [B], BVZ, [CEY], $\mathrm{Su}$ ] for a variety of results and points of view.

As is well-known, the spectrum has a very interesting dependence on $\theta$ : for rational values $\theta=\frac{p}{q}$, the spectrum is a union of $q+1$ intervals in $[-1,1]$ with $q$ gaps between them; when $\theta$ is irrational, the spectrum is often (and conjecturally always) a Cantor set. These facts can be proved by studying the $C^{*}$-algebra $A_{\theta}$ generated by these unitaries (the so-called rotation algebra, or non-commutative torus). See the articles cited above.

The Harper operator could be studied from the point of view of this paper as a discrete random walk on a Lie group (namely, $N_{\mathbb{R}}$ ). However, our concern will be with a compact analogue on the unitary group $U(q)$. Namely, for $\theta=\frac{p}{q}$ with 
$(p, q)=1$, the algebra $A_{\theta}$ has an irreducible representation $\pi$ taking $U$ to the diagonal matrix $u:=\operatorname{diag}\left(1, e^{i \theta}, \ldots, e^{(q-1) \theta}\right)$ and $V$ into the cyclic permutation operator $v$ on the standard basis $e_{2} \rightarrow e_{1}, \ldots, e_{n} \rightarrow e_{n-1}, e_{1} \rightarrow e_{n}$. These matrices are unitary, hence $\pi\left(H_{\theta}\right)$ is the transition operator of a random walk

$$
\mu_{\theta}:=\frac{1}{4}\left(\delta_{u}+\delta_{u^{*}}+\delta_{v}+\delta_{v^{*}}\right)
$$

on $U(q)$. Given a ray of representations $\mathbb{N} \rho$ of $U(q)$ we then get an example of a random walk $\left(U(q), \mu_{\theta}, H^{2}\left(B_{\rho}\right)\right)$ to which the Main Theorem applies.

The relation between the spectrum of the random walk $\mu_{\theta}$ and the spectrum of the Harper operator is not so immediate, however. The problem is that along the ray, $u$, resp. $v$, act by the translation operators $T_{u}$, resp. $T_{v}$, on $H^{2}\left(B_{\rho}\right)$. The commutation relation between $T_{u}$ and $T_{v}$ along the ray is then given by

$$
T_{u} T_{v}=T_{z} T_{v} T_{u}
$$

where $T_{z}$ is translation by $z=e^{i \theta}$. Evidently $T_{z}$ is not multiplication by a scalar so the algebra $\left\langle T_{u}, T_{v}\right\rangle$ generated by $T_{u}, T_{v}$ is not the rotation algebra.

Nevertheless, as will be explained in $\S 4$, the spectrum of $T_{\mu}$ along a ray can be related to the spectra of a finite number of Harper operators. Hence the analogy to the rational Harper operator is not too far fetched. From this example, we see that the spectrum of discrete random walks along rays of representations can have interesting multiple band-gap structures.

This article is intended for readers with an interest in random walks but with little or no background in microlocal analysis. For this reason, we have included rather lengthy expository sections summarizing the relevant material from homogeneous quantization theory and Fourier integral operators (Sections 1-2). Much of the proof of the theorem above will be given, as examples and illustrations, in the course of the exposition, so it should be regarded as an integral portion of the text. The microlocalization of a random walk to a ray of representations actually requires the theory of Toeplitz operators of [BdMG]. We will be building on this material as well in the sequel.

\section{ACKNOWLEDGEMENTS}

The discussion in $\S 4$ of "pseudo-Harper" operators has benefitted from discussions with Professor G.A. Elliott. Any remaining errors or oversights are of course the responsibility of the authors.

\section{REVIEW OF MiCROLOCAL ANALYSIS}

The purpose of this section is to present the microlocal machinery that we will need for the proof of the Main Theorem. Moreover, much of the proof will actually be carried out in the course of introducing the basic notions. Let us begin with some heuristic background.

Microlocal analysis is, among other things, a rigorous version of quantization theory and provides a table of correspondences between notions of classical and quantum mechanics. For the purposes of this paper, classical mechanics means the study of the dynamics of canonical transformations $f$ and Hamiltonian group actions $T_{g}$ on symplectic manifolds $(M, \omega)$. Quantum mechanics means the study of the spectral theory of their quantizations as unitary Fourier Integral operators $U_{f}$, resp. $U_{g}$, on an associated Hilbert space. 
Specifically, the classical mechanics will in part consist of the (Hamiltonian) action of $G$ by conjugation on the co-adjoint orbits $\mathcal{O}_{\rho}$ and on certain symplectic cones $Y$ encompassing all the orbits in the ray through $\rho$. But more interestingly it will consist in the dynamics of the random walk itself-i.e. the discrete dynamical system generated by the Markov operator $T_{\mu}$ - on these symplectic manifolds. The quantum mechanics will consist in part of the representation theory of $G$ on the Hilbert space corresponding to $\mathcal{O}_{\rho}$, namely $V_{\rho}$ and on the Hilbert space $H^{2}(B)$ corresponding to $Y$, the ray of representations $\bigoplus_{r=1}^{\infty} V_{r \rho}$ thru $\rho$. But the focus is on the spectrum of the Markov operator $T_{\mu}$ on the Hilbert spaces $V_{\rho}$ and $H^{2}(B)$.

1.1. Symplectic manifolds. Throughout the paper, we assume familiarity with basic notions from differential geometry. For background see, for example, [N]. Let $M$ be a compact connected manifold of dimension $n$. $M$ is called symplectic if $M$ possesses a symplectic form $\omega$, i.e., a nondegenerate closed $(d \omega=0)$ differential 2form. Note that the non-degeneracy of $\omega$ implies that $n$ be even. For each $x \in M$, the tangent space $T_{x} M$ at $x$ is isomorphic to the cotangent space $T_{x}^{*} M$ via the map

$$
v_{x} \rightarrow \iota\left(v_{x}\right) \omega_{x}
$$

where $\iota$ denotes the usual insertion map (here, $\iota\left(v_{x}\right) \omega_{x}=\omega_{x}\left(v_{x}, \cdot\right)$.

We now assume there exists a group action $G \times M \rightarrow M,(g, x) \rightarrow g \cdot x$ of the Lie group $G$ on the symplectic manifold $(M, \omega)$. For each $(g, x) \in G \times N$, the differential of the group action induces a linear map $T_{g} G \times T_{x} N \rightarrow T_{g \cdot x} M$. Take in particular $g=e$, the identity element of $G$. Then for each $X \in \mathbf{g}=T_{e} G$, the Lie algebra of $G$, we get a vector field $X^{\#}$ on $M$ (the image of $(X, 0)$ under this map). We call the group action symplectic if it preserves the symplectic structure, i.e., if

$$
D_{X \# \omega}=0 \quad\left(\Leftrightarrow d\left(\iota\left(X^{\#}\right) \omega=0\right) .\right.
$$

If for all $X \in \mathbf{g}$ the form $\left(\iota\left(X^{\#}\right) \omega\right.$ is exact, i.e., there exists a smooth function $\phi_{X}$ on $M$ such that $\iota\left(X^{\#}\right) \omega=d \phi_{X}$ (the $X^{\#}$ 's are symplectic gradient vector fields), we call the group action Hamiltonian. The corresponding moment map $\Phi: M \rightarrow \mathbf{g}^{*}$ (where $\mathbf{g}^{*}$ is the dual of the Lie algebra $\mathbf{g}$ ) is defined by

$$
\langle X, \Phi(x)\rangle=\phi_{X}(x) .
$$

$\left(\langle X, \xi\rangle\right.$ with $\xi \in \mathbf{g}^{*}$ and $X \in \mathbf{g}$ denotes the natural pairing $\xi(X)$.) The following two examples will be crucial.

Example 1. The cotangent bundle of a manifold. Let $M$ be a smooth manifold, $T^{*} M$ its cotangent bundle, and $\pi$ the natural projection $\pi: T^{*} M \rightarrow M$. $T^{*} M$ carries a natural 1-form, $\alpha$, given by

$$
\alpha_{(x, \xi)}=\xi \circ d \pi_{(x, \xi)} \quad\left(\Leftrightarrow \alpha_{(x, \xi)}=\pi^{*}(x, \xi)\right) .
$$

Here $(x, \xi)$ are the local coordinates of a point in $T_{x}^{*} M$ (short for $\left(x_{1}, \ldots, x_{n}\right.$, $\left.\left.\xi_{1}, \ldots, \xi_{n}\right)\right)$. In these local coordinates, $\alpha$ can be written as $\alpha=\sum_{i=1}^{n} \xi_{1} d x_{1}$. The canonical 2-form on $T_{x}^{*} M$ is $\omega=d \alpha\left(=\sum_{i=1}^{n} d \xi_{i} \wedge d x_{i}\right)$.

Example 2. Coadjoint orbits. Let $G$ be a Lie group, g its Lie algebra (which can be identified with the tangent space $T_{e} G$ at the identity $e \in G$ ), and $\mathbf{g}^{*}$ the dual of g. Recall that $G$ acts on $\mathbf{g}$ via the adjoint representation $\mathrm{Ad}$ :

$$
g \cdot X=\operatorname{Ad}(g) X:=d\left(c_{g}\right)_{e} X \quad \text { for all } X \in \mathbf{g}, g \in G
$$


where $c_{g}: G \rightarrow G$ is the conjugation map $c_{g}(h)=g h g^{-1}$ and $d\left(c_{g}\right)$ denotes its differential. Hence by duality we get the coadjoint action $\mathrm{Ad}^{*}$ of $G$ on $\mathbf{g}^{*}$ :

$$
\left\langle X, \operatorname{Ad}^{*}(g) \xi\right\rangle=\left\langle\operatorname{Ad}\left(g^{-1}\right) X, \xi\right\rangle
$$

for all $g \in G, X \in \mathbf{g}$, and $\xi \in \mathbf{g}^{*}$. For any $\xi \in \mathbf{g}^{*}$, the corresponding coadjoint orbit $\mathcal{O}_{\xi}=\left\{\operatorname{Ad}^{*}(g) \xi: g \in G\right\}$ is a symplectic manifold and the (coadjoint) action of $G$ on $\mathcal{O}_{\xi}$ is Hamiltonian. The symplectic structure is given by $\omega$ with

$$
\omega_{\eta}\left(X_{\eta}^{\#}, Y_{\eta}^{\#}\right)=\langle[X, Y], \eta\rangle \quad \text { for all } \eta \in \mathcal{O}_{\xi}
$$

and the moment map $\Phi: \mathcal{O}_{\xi} \rightarrow \mathbf{g}^{*}$ is the natural inclusion map $i$. See [Ki] for details and proofs.

Let $(M, \omega)$ and $\left(M^{\prime}, \omega^{\prime}\right)$ be two symplectic manifolds and $f: M \rightarrow M^{\prime}$ a diffeomorphism. $f$ is called a symplectomorphism or a canonical transformation if $f^{*} \omega^{\prime}=\omega$.

Example 3. The lift of a diffeomorphism. Let $X, X^{\prime}$ be two manifolds and $f$ : $X \rightarrow X^{\prime}$ a diffeomorphism. We can lift $f$ to a diffeomorphism $F: T^{*} X \rightarrow T^{*} X^{\prime}$ of the cotangent bundles by defining $F(x, \xi)=\left(f(x),\left(f^{-1}\right)^{*} \xi\right)$. This map $F$ is a canonical transformation with respect to the canonical 2 -forms $\omega$ and $\omega^{\prime}$ on $T^{*} X$ and $T^{*} X^{\prime}$, respectively.

1.2. Lagrangean submanifolds. Let $\Lambda \subset M$ be a smooth submanifold of the symplectic manifold $(M, \omega)$. For each $x \in \Lambda$, the tangent space $T_{x} \Lambda$ is a subspace of $T_{x} M$. We denote the orthogonal complement of $T_{x} \Lambda$ in $T_{x} M$ with respect to $\omega_{x}$ by $T_{x}^{\perp} \Lambda . \quad \Lambda$ is called isotropic if for all $x \in \Lambda, T_{x} \Lambda \subseteq T_{x}^{\perp} \Lambda$, i.e., $\omega 1_{\Lambda}=0$ ( $\omega 1_{\Lambda}=i^{*} \omega$ where $i: \Lambda \rightarrow M$ is the natural inclusion map). If $\Lambda$ is maximal isotropic, i.e., if $T_{x} \Lambda=T_{x}^{\perp} \Lambda$ for all $x \in \Lambda$, we call $\Lambda$ Lagrangean. An isotropic subspace $\Lambda$ is Lagrangean if and only if $\operatorname{dim} \Lambda=\frac{1}{2} \operatorname{dim} M$.

Let $\left(M_{1}, \omega_{1}\right)$ and $\left(M_{2}, \omega_{2}\right)$ be two symplectic manifolds. Clearly, $M_{2}^{\prime}=$ $\left(M_{2},-\omega_{2}\right), M=\left(M_{1} \times M_{2}, \omega_{1}+\omega_{2}\right)$, and $M^{\prime}=\left(M_{1} \times M_{2}, \omega_{1}-\omega_{2}\right)$ are also symplectic manifolds. We call a Lagrangean submanifold $\Lambda^{\prime} \subset M^{\prime}$ a canonical relation.

Example 4. Graphs of canonical transformations. Let $\left(M_{1}, \omega_{1}\right)$ and $\left(M_{2}, \omega_{2}\right)$ be two symplectic manifolds and $f: M_{1} \rightarrow M_{2}$ a canonical transformation. Since $f^{*} \omega_{2}=\omega_{1}, \omega_{1}-\omega_{2}$ vanishes on $\operatorname{Graph}(f)=\left\{\left(m_{1}, f\left(m_{1}\right)\right) \mid m_{1} \in M_{1}\right\} \cong M_{1}$. Furthermore, $\operatorname{dim} \operatorname{Graph}(f)=\operatorname{dim} M_{1}=\frac{1}{2} \operatorname{dim}\left(M_{1} \times M_{2}\right)$. Thus Graph $(f)$ is a Lagrangean submanifold of $M^{\prime}$.

Of particular relevance in this article are the homogeneous canonical transformations on cotangent bundles; that is, canonical transformations $\chi: T^{*} X \rightarrow T^{*} Y$ satisfying $\chi(x, r \xi)=r \chi(x, \xi)$ for $r>0$. The simplest examples are lifts $F$ to the cotangent bundle $T^{*} X$ of diffeomorphisms $f: X \rightarrow X$. The graph Graph $\chi$ of any homogeneous canonical transformation is naturally diffeomorphic to $T^{*} X$ under either of the projections

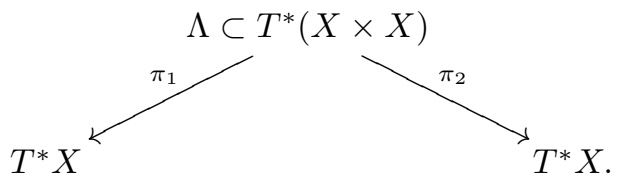


Example 5. The conormal bundle of a submanifold. Let $X$ be a manifold and $Y \subset X$ a smooth submanifold. The conormal bundle $N^{*} Y \subset T^{*} X$ is defined as the set

$$
N^{*} Y=\left\{(y, \xi) \mid y \in Y, \xi(v)=0 \text { for all } v \in T_{y} Y\right\} .
$$

We can define local coordinates $x_{1}, x_{2}, \ldots, x_{n}$ in $X$ such that $Y$ is given by $x_{1}=$ $x_{2}=\ldots=x_{k}=0$. Thus, with respect to the coordinates $x_{1}, \ldots, x_{n}, \xi_{1}, \ldots, \xi_{n}$ in $T^{*} X, N^{*} Y$ is defined by $x_{1}=\cdots=x_{k}=\xi_{k+1}, \cdots, \xi_{n}=0$ and of dimension $n$. It is clear that the natural 1-form $\alpha$ (see Example 1) is 0 on $T\left(N^{*} Y\right)$, therefore also the canonical 2-form $\omega=d \alpha$, and $N^{*} Y$ is a Lagrangean submanifold of $T^{*} X$.

A manifold $M$ is called conic, if there exists a free $\mathbb{R}^{+}$action on $M$. If $M_{1}, M_{2}$ and $\Lambda^{\prime}$ are all conic, we call $\Lambda^{\prime}$ a homogeneous canonical relation. Here, conic and homogeneous are synonyms. In this context, homogeneous has nothing to do with "homogeneous space".

Two canonical relations $\Lambda_{1}^{\prime} \subset M_{1} \times M_{2}$ and $\Lambda_{2}^{\prime} \subset M_{2} \times M_{3}$ can be composed to

$$
\Lambda^{\prime}=\Lambda_{1}^{\prime} \circ \Lambda_{2}^{\prime}=\left\{\left(\gamma_{1}, \gamma_{3}\right) \mid\left(\gamma_{1}, \gamma_{2}\right) \in \Lambda_{1} \text { and }\left(\gamma_{2}, \gamma_{3}\right) \in \Lambda_{2} \text { for some } \gamma_{2}\right\} .
$$

Equivalently, $\Lambda^{\prime}$ is the image of $\Lambda_{1}^{\prime} \times \Lambda_{2}^{\prime} \cap M_{1} \times \Delta\left(M_{2}\right) \times M_{3}$ under the projection

$$
\pi: M_{1} \times M_{2} \times M_{2} \times M_{3} \rightarrow M_{1} \times M_{3} .
$$

(Here $\Delta\left(M_{2}\right)$ denotes the diagonal $\left.\left\{\left(\gamma_{2}, \gamma_{2}\right) \mid \gamma_{2} \in M_{2}\right\}\right)$. In general, $\Lambda^{\prime}=\Lambda_{1}^{\prime} \circ \Lambda_{2}^{\prime}$ is not a canonical relation. However, a clean intersection hypothesis gives a sufficient condition. Before we state this result, let us record the following definition.

Definition 1.2.1. Let $X, Y, Z$ be smooth manifolds and $f: X \rightarrow Z$ and $g: Y \rightarrow Z$ two maps. The fiber product

$$
F=\{(x, y) \in X \times Y \mid f(x)=g(y)\}
$$

can be represented by the diagram

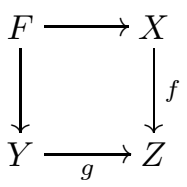

We say the two maps $f$ and $g$ intersect cleanly if

1) $F$ is a submanifold of $X \times Y$ and

2) for each $p \in F, p=(x, y)$, the tangent space $T_{p} F$ is the fiber product of the tangent spaces $T_{x} X$ and $T_{y} Y$, i.e., the diagram

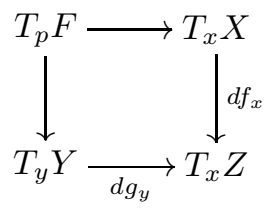

is a fiber diagram. The number $e=\operatorname{dim} F+\operatorname{dim} Z-(\operatorname{dim} X+\operatorname{dim} Y)$ is called the excess of the intersection. If $e=0$, the intersection is transversal.

For the following theorem see, for example, [DG, Lemma 5.3. 
Theorem 1.2.2. Let $\Lambda_{1}^{\prime} \subset M_{1} \times M_{2}$ and $\Lambda_{2}^{\prime} \subset M_{2} \times M_{3}$ be two canonical relations. If the fiber product diagram

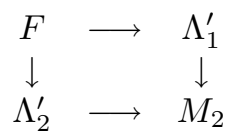

is clean, then $\Lambda^{\prime}:=\Lambda_{1}^{\prime} \circ \Lambda_{2}^{\prime}$ is an immersed canonical relation in $M_{1} \times M_{3}$ and the map

$$
\alpha: F \rightarrow \Lambda^{\prime}
$$

is a fiber mapping with compact fiber.

1.3. Lagrangean distributions and FIO's. These are distributions which can be written as certain types of oscillatory integrals $\int a e^{i \phi}$. We begin by describing the assumptions on the amplitude $a$ and the phase $\phi$. Let $M$ be an $n$-dimensional smooth manifold and $X \subset M$ an open set.

Definition 1.3.1. A function $\phi \in C^{\infty}\left(X \times(\mathbb{R} \backslash\{0\})^{N}\right)$ is called a phase function if

1) $\operatorname{Im} \phi \geq 0$

2) $\phi(x, r \theta)=r \phi(x, \theta)$ for all $r \in \mathbb{R}^{+}$,

3) $d \phi \neq 0$.

The phase function $\phi$ is called non-degenerate if from $d_{\theta} \phi(x, \theta)=0,(x, \theta) \in X \times$ $(\mathbb{R} \backslash\{0\})^{N}$, it follows that $d_{(x, \theta)} \frac{\partial \phi}{\partial \theta_{j}}(x, \theta)$ are linearly independent for $j=1, \ldots, N$.

It follows from the implicit function theorem that

$$
C_{\phi}=\left\{(x, \theta) \mid d_{\theta}(x, \theta)=0\right\},
$$

called the critical set of $\phi$, is a conic $n$-dimensional smooth submanifold of $X \times$ $(\mathbb{R} \backslash\{0\})^{N}$. Its image under the map

$$
I_{\phi}: C_{\phi} \rightarrow T^{*}(X), \quad I_{\phi}(x, \theta)=\left(x, d_{x} \phi(x, \theta)\right)
$$

is an immersed, conic, Lagrangean submanifold, $\Lambda_{\phi}$, of $T^{*}(X)$ (see $[\mathrm{D}$, Section 2.3). It can be shown (see GS3, Prop. 11.4) that vice versa, every closed conic Lagrangean submanifold $\Lambda \subset T^{*}(X) \backslash\{0\}$, can locally be parametrized in this way. More precisely, for every $\left(x_{0}, \theta_{0}\right) \in \Lambda$ there exists a non-degenerate phase function $\phi$ such that $\Lambda=\Lambda_{\phi}$ in a neighbourhood of $\left(x_{0}, \theta_{0}\right)$.

Definition 1.3.2. Let $m$ be a real number. The set $S^{m}\left(X \times \mathbb{R}^{N}\right)$, called the space of symbols of order $m$, is defined as the space of all $a \in C^{\infty}\left(X \times \mathbb{R}^{N}\right)$ with the property that for all compact $K \subset X$ and all $\alpha \in \mathbb{N}^{n}, \beta \in \mathbb{N}^{N}$, there exists a constant $C_{K, \alpha, \beta}(a)$ such that

$$
\left|\partial_{x}^{\alpha} \partial_{\theta}^{\beta} a(x, \theta)\right| \leq C(1+|\theta|)^{m-|\beta|}
$$

for all $(x, \theta) \in X \times \mathbb{R}^{N}$. A symbol $a \in S^{m}\left(X \times \mathbb{R}^{N}\right)$ has an asymptotic expansion

$$
a(x, \theta) \sim \sum_{j=0}^{\infty} a_{j}(x, \theta) \quad(\theta \rightarrow \infty)
$$


with $a_{j} \in S^{m-j}$. The space of polyhomogeneous or classical symbols of degree $m$, $S_{p h g}^{m}$, is the set of all symbols $a$ such that

$$
a(x, \theta) \sim \sum_{j=0}^{\infty} a_{j}(x, \theta)
$$

where $a_{j}$ is homogeneous of degree $m-j$, i.e., $a(x, r \theta)=r^{m-j} a(x, \theta)$ for $r>0$.

For a fixed phase function $\phi$ and a fixed symbol $a \in S^{m}\left(X \times \mathbb{R}^{N}\right)$, the oscillatory integral $I_{\phi}(a)$ given by

$$
I_{\phi}(a)=\int e^{i \phi(x, \theta)} a(x, \theta) d \theta
$$

defines a distribution, i.e., an element in $\mathcal{D}^{\prime}\left(X \times \mathbb{R}^{N}\right)$. Such a $u=I_{\phi}(a)$ has its singularities (its "wave front set") in $\Lambda_{\phi}$.

Definition 1.3.3. Let $\Lambda \subset T^{*}(X) \backslash\{0\}$ be a closed conic Lagrangean submanifold of $T^{*}(X)$. The space of Lagrangean distributions of order $m$ associated with $\Lambda$, $I^{m}(X, \Lambda)$, is defined as the space of all $u \in \mathcal{D}^{\prime}(X)$ with the following properties:

1) The wave front set $\operatorname{WF}(u) \subset \Lambda$.

2) For all $\left(x_{0}, \theta_{0}\right) \in \Lambda$ and any non-degenerate phase function $\phi$, defined on an open cone $V \subset X \times(\mathbb{R} \backslash\{0\})^{N}$ and parametrizing $\Lambda$ in a neighbourhood of $\left(x_{0}, \theta_{0}\right)$, there exists a symbol $a \in S^{m+\frac{n}{4}-\frac{N}{2}}\left(X \times \mathbb{R}^{N}\right)$ with support in a cone $\subset V$ such that $u \equiv I_{\phi}(a)$ in neighbourhood of $\left(x_{0}, \theta_{0}\right)$.

The local representation of a Lagrangean distribution in terms of an oscillatory integral is not unique. On the other hand, for a given Lagrangean distribution $u$, the Lagrangean $\Lambda$, its principal symbol $\sigma_{u}$, which we will describe below, and its order are uniquely determined. Our calculations will only involve these invariants.

Definition 1.3.4. If $u$ is a Lagrangean distribution on $X$ satisfying $u(1)=1$ and $u(f) \geq 0$ for all nonnegative $f \in C_{0}^{\infty}(X)$, then $u$ is called a Lagrangean probability measure.

Example 6. Discrete measures. The delta function $\delta_{x_{0}}(x)$ on $\mathbb{R}^{n}$ at a point $x_{0} \in$ $\mathbb{R}^{n}$ is given by the oscillatory integral

$$
\delta_{x_{0}}(x)=(2 \pi)^{-n} \int_{\mathbb{R}^{n}} e^{i\left\langle x-x_{0}, \theta\right\rangle} d \theta
$$

We have $\Lambda_{\phi}=T_{x_{0}}^{*} \mathbb{R}$ and $\delta_{x_{0}} \in I^{\frac{n}{4}}\left(\mathbb{R}, T_{x_{0}}^{*} \mathbb{R}\right)$. More generally, a discrete measure

$$
\mu=\frac{1}{m_{\mu}} \sum_{i=1}^{k} c_{i} \delta_{x_{i}}
$$

with $m_{\mu}=\sum_{i=1}^{k} c_{i}$ on a manifold $M$ of dimension $n$ is a mixture of delta functions, hence can be expressed in local coordinates as a sum of oscillatory integrals of the form (1.3.6). We have $\mu \in I^{\frac{n}{4}}(M, \Lambda)$ with $\Lambda=\bigcup_{x \in \operatorname{supp} \mu} T_{x}^{*} M$.

Example 7. Delta functions on submanifolds. Any $C^{\infty}$ density $u$ on a smooth submanifold $S \subset \mathbb{R}^{n}$ can locally be written as an oscillatory integral. Since $S$ is a smooth submanifold, for any given point $x_{0} \in S$ we can find $k$ functions $\phi_{1}, \ldots, \phi_{k}$ with $d \phi_{1}, \ldots, d \phi_{k}$ linearly independent at $x_{0}$, such that $S$ is defined in a neighbourhood around $x_{0}$ by

$$
\phi_{1}(x)=\ldots=\phi_{k}(x)=0 .
$$


There exists a function $a \in C_{0}^{\infty}\left(\mathbb{R}^{n}\right)$ with support near $x_{0}$ such that

$$
u(x)=(2 \pi)^{-k} \int_{\mathbb{R}^{k}} a(x) e^{i \phi(x, \theta)} d \theta \quad \text { where } \phi(x, \theta)=\sum_{j=1}^{k} \phi_{j}(x) \theta_{j} .
$$

Thus $\Lambda_{\phi}=\left\{\left(x, d_{x} \phi(x, \theta)\right) \mid \phi_{j}=0, j=1, \ldots, k, x \in \operatorname{supp} u\right\}$. Note that this is the conormal bundle $N^{*}(S)$ of $S$ restricted to supp $u$. We have $u \in I^{\frac{k}{2}-\frac{n}{4}}\left(\mathbb{R}^{n}, \Lambda_{\phi}\right)$.

Delta functions on submanifolds are examples of conormal distributions. They are defined as having a local expression as an oscillatory integral with phase function linear in $\theta$.

Example 8. Homogeneous distributions on the circle $S^{1}$. The basic homogeneous distributions on $S^{1}$ are the distributions $\chi_{k}(\theta)$ defined by the Fourier series

$$
\chi_{k}(\theta)=\sum_{r=1}^{\infty} r^{k} e^{i r \theta}
$$

with Fourier coefficients homogeneous of degree k. As Lagrangean distribution, $\chi_{k} \in I^{k+\frac{1}{4}}\left(S^{1}, T_{0}^{+*} S^{1}\right)$, that is, $\chi_{k}$ has only positive Fourier coefficients, has order $\left(k+\frac{1}{4}\right)$ and has sole singularity at $\theta=0$. It also has precisely the same singularity as the Fourier integral $(\theta+i 0)^{-k-1}:=\int_{0}^{\infty} \xi^{k} e^{i \theta \xi} d \xi$, i.e. differs from it in a neighborhood of 0 by a smooth function. (The integral needs to be regularized at $\xi=0$; we refer to [GS3] or to [Ho, I] for a more detailed discussion.) The analogous homogeneous distribution with singularity at $\theta=\theta_{0}$ instead of $\theta=0$ is given by the Fourier expansion

$$
\chi_{k}\left(\theta-\theta_{0}\right)=\sum_{r=1}^{\infty} r^{k} e^{i r \theta} e^{-i r \theta_{0}} .
$$

Example 9. Lagrangean distributions on $S^{1}$. Since $S^{1}$ is 1-dimensional, the homogeneous Lagrangean submanifolds of $T^{*} S^{1}$ must be finite unions of rays $T_{\theta_{j}}^{ \pm *}\left(S^{1}\right):=$ $\left\{\left(\theta_{j}, r \xi\right): r \pm 0\right\}$. Hence a Lagrangean distribution $\mathcal{Y}$ on $S^{1}$ can only have a finite number of singular points $\left\{\theta_{1}, \ldots, \theta_{n}\right\}$. Since $T_{\theta_{j}}^{ \pm *}\left(S^{1}\right)$ is parametrized by the phase function $\phi(\theta, \xi):=\left(\theta-\theta_{j}\right) \xi$, near $\theta_{j}, \mathcal{Y}$ must have the form

$$
\mathcal{Y}(\theta)=\int_{0}^{\infty} e^{\left(\theta-\theta_{j}\right) \xi} a_{j}(\theta, \xi) d \xi
$$

with polyhomogeneous amplitude of the form

$$
a_{j}(\theta, \xi) \sim \sum_{i=0}^{\infty} a_{m_{j}-i} \xi^{m_{j}-i}
$$

Comparing with Example 8, we see that

$$
\mathcal{Y}(\theta) \sim \sum_{j=1}^{n} \sum_{i=0}^{\infty} a_{m_{j}-i} \chi_{m_{j}-i}\left(\theta-\theta_{j}\right) .
$$

Thus, $\mathcal{Y}$ is a finite sum of polyhomogeneous distributions.

Let $X$ and $Y$ be two $C^{\infty}$ manifolds. A distribution $k \in \mathcal{D}^{\prime}(X \times Y)$ defines a linear operator

$$
K: C_{0}^{\infty}(Y) \rightarrow \mathcal{D}^{\prime}(X)
$$


via

$$
\langle K(f), g\rangle=\langle k, f \otimes g\rangle, \quad f \in C_{0}^{\infty}(Y), g \in C_{0}^{\infty}(X) .
$$

Conversely, by the Schwartz kernel theorem, for every map $K$ as in (1.3.11) there exists a unique distribution $k \in \mathcal{D}^{\prime}(X \times Y)$ such that (1.3.12) is valid.

Definition 1.3.5. A linear operator $K: C_{0}^{\infty}(Y) \rightarrow \mathcal{D}^{\prime}(X)$ is called Fourier Integral Operator (FIO) if its Schwartz kernel $k$ is a Lagrangean distribution on $X \times Y$, i.e., if $k \in I^{m}(X \times Y, \Lambda)$ for some $m \in \mathbb{R}$ and $\Lambda \subset T^{*}(X \times Y)$ a Lagrangean submanifold. We will sometimes also write in this case, a bit loosely, $K \in I^{m}(X \times Y, \Lambda)$.

Notation. If $\Lambda \subset T^{*}(X \times Y)$ is a Lagrangean submanifold, we will denote $\{(x, y ; \xi, \eta) \mid(x, y ; \xi,-\eta) \in \Lambda\}$ by $\Lambda^{\prime}$. Recall that $\Lambda^{\prime}$ is a Lagrangean submanifold of $T^{*}(X \times Y)$ with respect to the symplectic form $\omega_{X}-\omega_{Y}$ where $\omega_{X}$ and $\omega_{Y}$ are the canonical forms on $T^{*} X$ and $T^{*} Y$, respectively.

The following clean composition theorem gives a sufficient condition for the composition of two FIO's to be FIO again. See, for example, Ho, IV, Theorem 25.2.3].

Theorem 1.3.6. Let $X, Y, Z$ be smooth manifolds and $\Lambda_{1}^{\prime} \subset T^{*} X \times T^{*} Y$ and $\Lambda_{2}^{\prime} \subset$ $T^{*} Y \times T^{*} Z$ two homogeneous canonical relations. Let $K_{1}$ and $K_{2}$ be two FIO's whose Schwartz kernels $k_{1}$ and $k_{2}$ are in $I^{m_{1}}\left(X \times Y, \Lambda_{1}\right)$ and $I^{m_{2}}\left(Y \times Z, \Lambda_{2}\right)$, respectively. Assume that the composition $\Lambda=\Lambda_{1}^{\prime} \circ \Lambda_{2}$ is clean with excess $e$. Then $K_{3}=K_{1} K_{2}$ is also an FIO and its Schwartz kernel

$$
k_{3} \in I^{m_{1}+m_{2}+e / 2}(X \times Z, \Lambda) .
$$

With few exceptions (the Szegö projector and the trace operation), all of the Fourier integral operators (FIO's) we will be considering in this paper come from Fourier integral representations of Lie groups. In fact, they will just be translations by group elements on homogeneous spaces. The following gives a summary of the notation and terminology regarding FIO group actions.

1.4. Hamiltonian and Fourier integral group actions. We begin by describing the simplest Hamiltonian actions on the cotangent bundle $T^{*} M$ of a compact manifold $M$ which arise from lifts of geometric (translation) actions on the base $M$.

Thus, let $l: G \times M \rightarrow M$ be an action of the Lie group $G$ on $M$. The action of $G$ on $M$ can be lifted to an action $L$ of $G$ on the cotangent bundle $T^{*} M$ in a canonical way: For fixed $g \in G, L(g ; m, \xi)=\left(l_{g}(m),\left(d l_{g^{-1}}\right)^{*} \xi\right)$ ) for all $(m, \xi) \in T^{*} M$ (we have denoted $l(g, m)$ by $\left.l_{g}(m)\right)$. This action is always Hamiltonian and we denote its moment map by $\Phi$. It is determined by the following formula:

$$
\iota\left(X^{\#}\right) \omega=d(\langle X, \Phi(m, \xi)\rangle) \quad \text { for all } X \in \mathbf{g},(m, \xi) \in T^{*} M .
$$

More concretely, let $\left\{X_{1}, \ldots, X_{r}\right\}$ denote a basis of the Lie algebra $\mathbf{g}$ of $G$ and let $X_{i}^{\#}$ denote the corresponding vector fields on $M$. They are all Hamiltonian vector fields on $T^{*} M$ with Hamiltonians $f_{i}(x, \xi):=\left\langle\left(X_{i}^{\#}\right)_{x}, \xi\right\rangle$. The moment map can then be written as

$$
\Phi(x, \xi)=\left(f_{1}, \ldots, f_{r}\right) .
$$

In particular if $G=S^{1}$, and if $\frac{\partial}{\partial \theta}$ denotes the generator of the action on $M$, then the moment map is the Hamiltonian $f(x, \xi):=\left\langle\frac{\partial}{\partial \theta}, \xi\right\rangle$.

The action $l$ of $G$ on $M$ induces a unitary representation $\rho$

$$
\rho(g) \cdot \eta=l_{g}^{*} \eta
$$


of $G$ on $L^{2}\left(M, \Omega^{\frac{1}{2}}\right)$, the space of half densities on $M$. Here, a $1 / 2$-density $\eta$ is the square root $|d v o l|^{1 / 2}$ of a volume density on $M$. These rather unusual objects give $M$ an intrisnic $L^{2}$-structure since the product of two $1 / 2$-densities is a density which can be integrated over $M$. They are ubiquitous in microlocal analysis and although all of our manifolds have natural volume densities, we will state things in terms of $1 / 2$-densities to enable us easily to quote relevant microlocal facts from the references.

Since $\rho(g)$ is translation (pull-back) by $g$, the Schwartz kernel $k_{g}(x, y)$ of $\rho(g)$ is essentially the delta-function $\delta\left(y-l_{g}(x)\right)$. To make this intuitively clear notion precise, we would have to discuss delta $1 / 2$-densities on a manifold. For the sake of brevity, we refer to $\mathrm{G}$ for the precise definitions, and only note that as a delta function on $\operatorname{Graph}(l)=\{(g, m, l(g, m)) \mid g \in G, m \in M\}, k(g, x, y)$ is a Lagrangean distribution on $G \times M \times M$ with associated Lagrangean $\Gamma$ equal to the conormal bundle of $\operatorname{Graph}(l)$, which is easily seen to equal the moment Lagrangian of the Hamiltonian action of $G$ on $T^{*} M$, given by

$$
\Gamma=\left\{(g, \Phi(m, \xi) ; m, \xi ; L(g ; m, \xi)) \mid g \in G,(m, \xi) \in T^{*} M\right\} .
$$

In the case of composition of FIO's coming from translations by (possibly different) group actions, the composition of FIO's is simply the composition of the translations and the composition of the Lagrangeans is simply given by the graph of the composite translation. For future reference, we consider this case in detail.

Cleanness of composition of $T_{g}$ and $T_{e^{i \theta}}$. Let $M$ be a manifold and $l: G \times M \rightarrow$ $M$ and and $k: S^{1} \times M \rightarrow M$ two group actions with $G$ not necessarily equal to $S^{1}$. For fixed $g \in G$, we denote the corresponding translation operator in $L^{2}\left(M, \Omega^{\frac{1}{2}}\right)$ by $T_{g}$. Its Schwartz kernel, $k_{g}(x, y)$, is a Lagrangean distribution (the delta function $\left.\delta\left(y-l_{g}(x)\right)\right)$ with corresponding Lagrangean

$$
\Lambda_{g}=\left\{\left(x, \xi ; l_{g}(x),-\left(d l_{g^{-1}}\right)^{*} \xi\right) \mid(x, \xi) \in T^{*} M\right\} \cong T^{*} M .
$$

For the group action $k: S^{1} \times M \rightarrow M$ we also consider translation in $L^{2}\left(M, \Omega^{\frac{1}{2}}\right)$, denoted by $T_{e^{i \theta}}$. Now we do not hold a specific element in $S^{1}$ fixed, but view the resulting representation of $S^{1}$ as a distribution on $S^{1} \times M \times M$. This distribution is a delta function on

$$
\operatorname{Graph}(k)=\left\{\left(e^{i \theta} ; x ; k\left(e^{i \theta}, x\right)\right) \mid e^{i \theta} \in S^{1}, x \in M\right\}
$$

whose conormal bundle is given by

$$
\Gamma=\left\{\left(e^{i \theta}, \Phi(x, \xi) ; x, \xi ; K_{\theta}(x, \xi) \mid e^{i \theta} \in S^{1},(x, \xi) \in T^{*} M\right\} \cong S^{1} \times T^{*} M .\right.
$$

Here $K_{\theta}(x, \xi):=\left(k\left(e^{i \theta}, x\right), d k_{e^{-i \theta}}^{*} \xi\right)$ and $\Phi$ denotes the moment map of the group action $K: S^{1} \times T^{*} M \rightarrow T^{*} M$.

The Schwartz kernel of the operator $T_{e^{i \theta} g}:=T_{e^{i \theta}} \circ T_{g}$, viewed as a distribution on $S^{1} \times B \times B$, is always Lagrangean, since the composition $\Gamma^{\prime} \circ \Lambda_{g}^{\prime}$ is automatically clean (see Theorem 1.3.6). We recall that this means that the fiber diagram

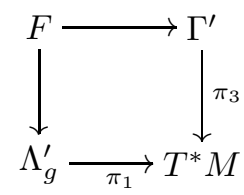


is clean, where $\pi_{3}: \Lambda^{\prime} \rightarrow T^{*} M$ denotes projection onto the third factor in $\Gamma^{\prime} \subset$ $T^{*} S^{1} \times T^{*} M \times T^{*} M, \pi_{1}: \Lambda_{g}^{\prime} \rightarrow T^{*} M$ is projection onto the first factor, and where

$$
\begin{aligned}
F & =\left\{\left(e^{i \theta}, \Phi(x, \xi) ; x, \xi ; K_{\theta}(x, \xi) ; K_{\theta}(x, \xi) ; L_{g} K_{\theta}(x, \xi)\right) \mid e^{i \theta} \in S^{1},(x, \xi) \in T^{*} M\right\} \\
& \cong S^{1} \times T^{*} M
\end{aligned}
$$

with $L_{g}(x, \xi):=\left(l_{g}(x),\left(d l_{g^{-1}}\right)^{*} \xi\right)$. Since it is a graph, the fiber product $F$ is always a submanifold of $\Gamma^{\prime} \times \Lambda_{g}^{\prime}$ and it is easy to see that the derived diagram

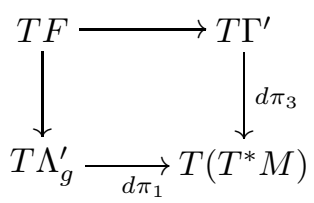

is a fiber diagram with excess $e=0$. Denoting the Lagrangean $\Gamma^{\prime} \circ \Lambda_{g}$ by $\Lambda$ we have

$$
\Lambda=\left\{\left(e^{i \theta}, \Phi(x, \xi) ; x, \xi ; L_{g} K_{\theta}(x, \xi)\right) \mid e^{i \theta} \in S^{1},(x, \xi) \in T^{*} M\right\} \cong S^{1} \times T^{*} M
$$

and

$$
T_{e^{i \theta} g} \in I^{-\frac{1}{4}}\left(S^{1} \times M \times M, \Lambda\right) .
$$

1.5. The trace operation. Taking the trace of an operator $A$ can be viewed as composing a certain Fourier integral operator Tr with the Schwartz kernel $k_{A}$ of $A$.

Let $k_{A}$ be a Lagrangean distribution on $G \times M \times M$. Then $\operatorname{Tr} A=\operatorname{Tr} k_{A}=$ $\int k_{A}(g, m, m) d m$. It was pointed out in [DG] that $\operatorname{Tr} A$ can be viewed as the composition

$$
\operatorname{Tr} A=\pi_{*} \Delta^{*} k_{A}
$$

with $\Delta: G \times M \rightarrow G \times M \times M$ the diagonal embedding and $\pi: G \times M \rightarrow G$ the projection onto the first factor.

Since the operator $\pi_{*} \Delta^{*}$ maps distributions on $G \times M \times M$ to distributions on $G$, its Schwartz kernel $k_{\pi_{*} \Delta^{*}}$ is a distribution on $G \times M \times M \times G$. The following lemma can be found in [DG], p. 66.

Lemma 1.5.1. We identify $G \times M \times M \times G$ with $(G \times M) \times(G \times M)$. The Schwartz kernel $k_{\pi_{*} \Delta^{*}}$ is the same as $k_{i d}$, the Schwartz kernel of the identity map. Furthermore, $k_{\pi_{*} \Delta^{*}} \in I^{0}(G \times M \times M \times G, \Gamma)$ with $\Gamma$ being the conormal bundle to the diagonal in $G \times M \times M \times G$.

1.6. The principal symbol of a Lagrangean distribution. Roughly speaking, the principal symbol $\sigma_{u}$ is a $1 / 2$-density on $\Lambda$. (To be completely accurate, it involves additional factors $e^{ \pm i \pi}$, called Maslov factors.) To define $\sigma_{u}$, we use a parametrization of $\Lambda$.

Let $u$ be an element in $I^{m}(X, \Lambda)$, and let

$$
I_{\phi}(a)=\int e^{i \phi} a d \theta
$$

be a local representation of $u$. We assume that the phase function $\phi u$ is defined in an open conic subset $V \subset X \times(\mathbb{R} \backslash\{0\})^{N}$ and that the projection of $V$ into $X$ is a domain of local coordinates $x_{1}, \ldots, x_{n}$. We can use $\partial \phi / \partial \theta_{i}, i=1, \ldots, N$, as local coordinates transversal to $C_{\phi}$ in $X \times(\mathbb{R} \backslash\{0\})^{N}$. On $C_{\phi}$ itself we choose local coordinates $c_{j}, j=1, \ldots, n$, that are homogeneous of degree one with respect to $\theta$. 
Together, the $\partial \phi / \partial \theta_{i}$ and $c_{j}$ form a complete coordinate system in $V . C_{\phi}$ carries a volume form $d_{\phi}$ defined by

$$
d_{\phi}=\left|\frac{D(x, \theta)}{D(c, \partial \phi / \partial \theta)}\right| d c_{1} \wedge \cdots \wedge d c_{n} .
$$

The coefficient on the right hand side denotes the Jacobian determinant of the $x_{j}$ and $\theta_{i}$ with respect to the $c_{j}$ and $\partial \phi / \partial \theta_{i}, j=1, \ldots, n$ and $i=1, \ldots, N$. Let

$$
a \sim a_{0}+a_{-1}+\ldots
$$

be the polyhomogeneous expansion of $a$ with $a_{0} \in S^{m+\frac{n}{4}-\frac{N}{2}}$ and with terms decreasing by steps of degree one. Then the local formula for the symbol is given by

$$
\sigma_{u}=a_{0} \sqrt{d_{\phi}}
$$

on $C_{\phi}$. More precisely, the diffeomorphism $I_{\phi}$ transfers the $1 / 2$-density $a_{0} \sqrt{d_{\phi}}$ on $C_{\phi}$ to a $1 / 2$-density $I_{\phi *} a_{0} \sqrt{d_{\phi}}$ on an open set $U \subset \Lambda$, which is by definition the symbol $\sigma_{u}$ on this set. Recall that the $c_{j}$ are homogeneous of degree one in $\theta$, the $\partial^{2} \phi / \partial \theta_{i} \partial \theta_{k}$ are of degree -1 in $\theta$, therefore $\frac{D(c, \partial \phi / \partial \theta)}{D(x, \theta)}$ is of degree $n-N$ in $\theta$ and the right hand side in (1.6.1) is of degree $N$ in $\theta$. It follows that $\sigma_{u} 1_{U} \in S^{m+\frac{n}{4}}\left(U ; \Omega^{\frac{1}{2}}\right)$ (here $\Omega^{\frac{1}{2}}$ denotes the half density bundle over $\Lambda$ ).

There is a complex line bundle, $\mathbb{L}$, over $\Lambda$, called the Keller-Maslov line bundle, which is also involved in piecing together the $\sigma$ from different coordinate patches in a smooth way. The precise definition of $\mathbb{L}$ is rather technical. Since it will play a minor role in our computations, we omit further details and refer the reader to [T], Chapters VII and VIII. Here we merely state the following.

Definition 1.6.1. The section $\sigma_{u} \in S^{m+\frac{n}{4}}\left(\Lambda ; \Omega^{\frac{1}{2}} \otimes \mathbb{L}\right)$ for which

$$
\sigma_{u} 1_{\Lambda_{\phi}}=I_{\phi *} a_{0} \sqrt{d_{\phi}}
$$

is called the principal symbol of $u$. The principal symbol $\sigma_{A}$ of a Fourier integral operator $A$ is the principal symbol of its Schwartz kernel $k_{A}$.

Example 10. The principal symbol of $T_{g}$. Recall that the Schwartz kernel of $T_{g}$, $k_{g}(x, y)$, is a Lagrangean distribution (the delta function $\left.\delta\left(y-l_{g}(x)\right)\right)$ with corresponding Lagrangean

$$
\Lambda=\left\{\left(x, \xi ; l_{g}(x),-\left(d l_{g^{-1}}\right)^{*} \xi\right) \mid(x, \xi) \in T^{*} M\right\} \cong T^{*} M .
$$

Any symplectic manifold $(N, \omega)$ of dimension $(2 n)$ carries a canonical volume form $\Omega:=(-1)^{n} \frac{1}{n !} \omega^{\wedge n}$. In the case of $T^{*} M, \Omega=d x_{1} \wedge \cdots \wedge d x_{n} \wedge d \xi_{1} \wedge \cdots \wedge d \xi_{n}$ for which we will write for brevity $d x \wedge d \xi$. Since $\Lambda$ is a graph, it carries the volume form $\pi^{*} \Omega$ where $\pi: \Lambda \rightarrow T^{*} M$ denotes projection onto the first factor. We claim that the principal symbol $\sigma_{T_{g}}$ is simply $\pi^{*}|\Omega|^{\frac{1}{2}}$. Indeed, for any coordinate patch $X \subset M$ we can write

$$
k_{g}(x, y) 1_{X \times X}=(2 \pi)^{-n} \int_{\mathbb{R}^{n}} e^{i \phi(x, y, \xi)} d \xi=(2 \pi)^{-n} \int_{\mathbb{R}^{n}} e^{i\left\langle\xi, y-l_{g}(x)\right\rangle} d \xi
$$

(recall Example 7). Since $\phi(x, y, \xi)=\left\langle\xi, y-l_{g}(x)\right\rangle$,

$$
C_{\phi}=\left\{\left(x, l_{g}(x), \xi \mid \xi \in \mathbb{R}^{n}\right\} \cong X \times \mathbb{R}^{n} \cong T^{*} X\right.
$$


and a complete coordinate system for $V=X \times X \times \mathbb{R}^{n}$ satisfying the above conditions is

$$
\begin{gathered}
c_{i}=\xi_{i}, \quad i=1, \ldots, n ; \quad c_{j}=x_{j}|\xi|, \quad j=n+1, \ldots, 2 n \\
\partial \phi / \partial \xi_{k}=y_{k}-\left(l_{g}(x)\right)_{k}, \quad k=1, \ldots, n .
\end{gathered}
$$

The inverse of the Jacobian in (1.6.1) is

$$
\left|\frac{D\left(\xi, x|\xi|, y-l_{g}(x)\right)}{D(x, y, \xi)}\right|=\left|\begin{array}{ccc}
0 & 0 & I \\
|\xi| I & 0 & \left(x_{i} \xi_{j} /|\xi|\right)_{1 \leq i, j \leq n} \\
d g & I & 0
\end{array}\right|=|\xi|^{n}
$$

(all blocks in the second determinant are of size $n \times n$ ). This yields for $d_{\phi}$ in (1.6.1),

$$
d_{\phi}=|\xi|^{-n} d c_{1} \wedge \cdots \wedge d c_{2 n}=d x \wedge d \xi=\Omega
$$

Since $a_{0}=1$ and clearly $I_{\phi}=\pi^{-1} 1_{T^{*} X}$, we have $I_{\phi \star}|\Omega|^{\frac{1}{2}}=\pi^{*}|\Omega|^{\frac{1}{2}}$ which proves that $\sigma_{T_{g}}=\pi^{*}|\Omega|^{\frac{1}{2}}$.

Example 11. The canonical 1/2-density on a graph. Generalizing Example 10, we note that the graph of any homogeneous canonical transformation $\chi$ carries a canonical 1/2-density. Since the graph of $\chi: T^{*} X \rightarrow T^{*} X$ is naturally diffeomorphic to $T^{*} X$ (by the conic diffeomorphism $\pi_{1}$, see $(1.2 .1)$ ), one can pull back the symplectic volume $1 / 2$-density $|\Omega|^{\frac{1}{2}}$ to the $1 / 2$-density $\pi_{1}^{*}|\Omega|^{\frac{1}{2}}=\pi_{1}^{*}|d x \wedge d \xi|^{\frac{1}{2}}$ on $\operatorname{Graph}(\chi)$. We will refer to it as the canonical graph $1 / 2$-density and denote it by $\left|\Omega_{\chi}\right|^{\frac{1}{2}}$. The calculation in Example 10 shows that $\sigma_{T_{g}}=\left|\Omega_{L_{g}}\right|^{\frac{1}{2}}$.

Example 12(a). The principal symbol of a discrete measure $\mu:=\sum_{j=1}^{k} c_{j} \delta_{x_{j}}$ on $\mathbb{R}$. Recall from Example 6 that $\mu \in I^{\frac{1}{4}}\left(\mathbb{R}, \bigcup_{j=1}^{k} T_{x_{j}}^{*} \mathbb{R}\right)$. Each cotangent space $T_{x_{j}}^{*} \mathbb{R} \cong \mathbb{R}$ carries a natural volume density $d t$. The principal symbol of $\mu$ is the $1 / 2$-density $\sigma_{\mu}$ on $\bigcup_{j=1}^{k} T_{x_{j}}^{*} \mathbb{R}$ with $\sigma_{\mu} 1_{T_{x_{j}}^{*} \mathbb{R}}=c_{j}|d t|^{\frac{1}{2}}$.

Example 12(b). The principal symbol of a transition operator $T:=\sum_{j=1}^{k} c_{j} T_{g_{j}}$. The Lagrangean corresponding to the Schwartz kernel $k_{T}$ of $T$ is the union $\Sigma=$ $\bigcup_{j=1}^{k} \Lambda_{g_{j}}$ where the $\Lambda_{g_{j}}$ 's are as in (1.4.4). By Example 10, the principal symbol of $T$ is the $1 / 2$-density $\sigma_{T}$ on $\Sigma$ with $\sigma_{T} 1_{\Lambda_{g_{j}}}=c_{j} \pi^{*}|\Omega|^{\frac{1}{2}}$.

Example 13. The principal symbol of a Fourier Integral group action. We return to the example of the moment Lagrangean $\Gamma$ (1.4.5) of a Hamiltonian $S^{1}$-action and to the associated Fourier integral representation $K_{\theta}$. The Lagrangean has a natural parametrization

$j: S^{1} \times T^{*} M \rightarrow T^{*} S^{1} \times T^{*} M \times T^{*} M \quad\left(e^{i \theta}, x, \xi\right) \rightarrow\left(e^{i \theta}, \Phi(x, \xi), x, \xi, K_{\theta}(x, \xi)\right)$

with image $\Gamma$. The parameter space carries the natural $1 / 2$-density $|d \theta|^{\frac{1}{2}} \otimes|\Omega|^{\frac{1}{2}}$ which gets transported to a $1 / 2$-density $\left|\Omega_{k}\right|^{\frac{1}{2}}$ on $\Gamma$, which we will refer to as the 'canonical density' on the graph of the action.

Proposition 1.6.2. The principal symbol $\sigma_{K}$ of a Fourier integral $S^{1}$-action is given by: $\sigma_{K}=\left|\Omega_{k}\right|^{\frac{1}{2}}$. 
Proof. (Sketch) Let us denote by $D$ the generator of the $S^{1}$-action, so that $K=$ $e^{i \theta D}$. Then $K$ is the unique solution of the inital value problem

$$
\left(\begin{array}{c}
\left(\frac{\partial}{\partial \theta}-D\right) K_{\theta}=0 \\
\left.K_{\theta}\right|_{\theta=0}=I d
\end{array}\right)
$$

The symbol can be determined by considering the corresponding initial value problem for the symbols. At $\theta=0$ we have the canonical graph $1 / 2$-density of the identity graph, and by the equation the symbol is invariant under the classical Hamiltonian $S^{1}$ action. The 1/2-density given in the statement of the proposition is the unique solution of this initial value problem. For more details, see [DG].

Example 14. The principal symbol of the trace operation. Recall from Lemma 1.5.1 that the Lagrangean $\Gamma$ corresponding to the Lagrangean distribution $k_{\pi_{\star}} \Delta_{\star}$ is the conormal bundle

$$
\Gamma=\left\{(g, \gamma ; x, \xi ; g,-\gamma ; x,-\xi) \mid(g, \gamma) \in T^{*} G,(x, \xi) \in T^{*} M\right\}
$$

of the diagonal $\Delta(G \times M)$. Clearly, $\Gamma \cong T^{*} G \times T^{*} M$. The principal symbol of the trace operation is

$$
\sigma_{T r}=\tilde{\pi}^{*}|d g \wedge d \gamma \wedge d x \wedge d \xi|^{\frac{1}{2}}
$$

where $\tilde{\pi}: \Gamma \rightarrow T^{*} G \times T^{*} M$ denotes projection onto the first and second factors. See, for example, Lemma 6.3 in DG].

We will also need to review the principal symbol calculus of FIO's. Given a pair of Lagrangeans $\Lambda_{1}$, respectively $\Lambda_{2}$, and a pair of $1 / 2$-densities $\sigma_{1}$, respectively $\sigma_{2}$, on them we wish to define a composite $1 / 2$-density $\sigma_{1} \circ \sigma_{2}$ on the composite Lagrangean $\Lambda_{1} \circ \Lambda_{2}$. To do so, we begin with the exterior tensor product of the $1 / 2$-densities, that is, the $1 / 2$-density on the product space

$$
\sigma_{1} \otimes \sigma_{2} \in \Omega^{\frac{1}{2}}\left(\Lambda_{1} \times \Lambda_{2}\right)
$$

given by

$$
\sigma_{1} \otimes \sigma_{2}\left(b_{1}, b_{2}\right):=\sigma_{1}\left(b_{1}\right) \sigma_{2}\left(b_{2}\right)
$$

where $b_{i}$ is a basis for $T_{\lambda_{i}}\left(\Lambda_{i}\right)$. The composition product is a bilinear operation

$$
\sigma_{1} \otimes \sigma_{2} \rightarrow \sigma_{1} \circ \sigma_{2}
$$

constructing $\sigma_{1} \circ \sigma_{2}$ in a canonical way from $\sigma_{1} \otimes \sigma_{2}$ in the form

$$
\left(\sigma_{1} \otimes \sigma_{2}\right)_{\lambda}=\int_{F_{\lambda}} \sigma_{1} \times \sigma_{2}
$$

with $\lambda \in \Lambda_{1} \circ \Lambda_{2}$, with $F_{\lambda}$ is the fiber $\left\{\left(\lambda_{1}, \lambda_{2}\right) \in \Lambda_{1} \times \Lambda_{2}: \lambda_{1} \circ \lambda_{2}=\lambda\right\}$ of the composition over $\lambda$, and with $\sigma_{1} \times \sigma_{2}$ a certain density along the fiber with values in $\Omega^{\frac{1}{2}}\left(\Lambda_{1} \circ \Lambda_{2}\right)$, the space of $1 / 2$-densities on the composite. See [Ho, IV] for the precise definition. Roughly speaking, if $\sigma_{1}=\sqrt{d x d z}$ and if $\sigma_{2}=\sqrt{d z d y}$, with $d z$ a volume density on $F_{\lambda}$, then $\sigma_{1} \times \sigma_{2}=\sqrt{d x d y} d z$

We will only need the following special cases of the composition law, which follow from the general composition theorem in [Ho, IV].

Theorem 1.6.3 (addendum). (a) Let $\Lambda_{i}=\operatorname{Graph}\left(\chi_{i}\right)$ be the graph of a homogeneous canonical transformation $\chi_{i}$ on $T^{*} M(i=1,2)$, and as above let $\left|\Omega_{\chi_{i}}\right|^{\frac{1}{2}}$ be the canonical 1/2-densities on the graphs. Then $\Lambda_{1} \circ \Lambda_{2}=\operatorname{Graph}\left(\chi_{1} \circ \chi_{2}\right)$ and $\left|\Omega_{\chi_{1}}\right|^{\frac{1}{2}} \circ\left|\Omega_{\chi_{2}}\right|^{\frac{1}{2}}=\left|\Omega_{\chi_{1} \circ \chi_{2}}\right|^{\frac{1}{2}}$. 
(b) Let $\Gamma$ be the moment Lagrangean of a classical Hamiltonian $S^{1}$ action $k$, equipped with the canonical density $\left|\Omega_{k}\right|^{\frac{1}{2}}$ and let $\operatorname{Graph}(\chi)$ be the graph of a homogeneous canonical transformation, equipped with the canonical graph 1/2-density $\left|\Omega_{\chi}\right|^{\frac{1}{2}}$. Then the composite Lagrangean $\Lambda$ (1.4.6) is parametrized by the map

$j_{\chi}: S^{1} \times T^{*} M \rightarrow T^{*} S^{1} \times T^{*} M \times T^{*} M \quad\left(e^{i \theta}, x, \xi\right) \rightarrow\left(e^{i \theta}, \Phi(x, \xi), x, \xi, L_{g} \chi(x, \xi)\right)$

and the composite 1/2-density is again given by $\left|\Omega_{\chi \circ k}\right|^{\frac{1}{2}}:=|d \theta|^{\frac{1}{2}} \otimes|\Omega|^{\frac{1}{2}}$.

\section{RAYS OF REPRESENTATIONS}

2.1. Geometric quantization. Let $G$ be a compact, semisimple Lie group, $\hat{G}$ its unitary dual, and $I^{*} \cap \mathbf{t}_{+}^{*}$ the set of lattice points in the chosen positive Weyl chamber $\mathbf{t}_{+}^{*}$. Recall that there is a one-to-one correspondencce $\hat{G} \leftrightarrow I^{*} \cap \mathbf{t}_{+}^{*}$ under which $\rho_{\nu} \leftrightarrow \nu$ with $\nu$ the highest weight of the irreducible character $\chi_{\rho_{\nu}}$. We call a representation $\rho$ of $G$ a ray of representations through $\nu$ if each irreducible $\rho_{n \nu}$, $n \in \mathbb{N}^{0}$ occurs exactly with multiplicity 1 in $\rho$.

Let $\mathcal{O}_{\nu}$ be the coadjoint orbit corresponding to the integral form $\nu \in I^{*} \cap \mathbf{t}_{+}^{*}$ (see Example 2 above). For any $\xi \in \mathcal{O}_{\nu}$, we denote the stabilizer group of $\xi$ with respect to the $G$-action by $G_{\xi}$. We have $\mathcal{O}_{\nu} \cong G / G_{\nu}$. Since $\nu$ is an integral point, the map $\chi_{\nu}: G_{\nu} \rightarrow S^{1}, g \rightarrow e^{2 \pi i\left\langle\nu, X_{g}\right\rangle}$ where $g=\exp X_{g}$ is a homomorphism (see GS1, (2.11)).

By the classical Borel-Weil-Bott theorem, there exists a holomorphic structure on the orbit $\mathcal{O}_{\nu}$ and a complex line bundle $E$ over $\mathcal{O}_{\nu}$ whose holomorphic sections $\Gamma(E)$ form the representation space of the irreducible representation of highest weight $\nu$. Let us explain the situation in more detail. The holomorphic line bundle $E$ is the quotient space of $G \times \mathbb{C}$ with respect to the equivalence relation

$$
(g h, z) \sim\left(g, \chi_{\nu}(h) z\right) \quad g \in G, h \in G_{\nu}, z \in \mathbb{C},
$$

i.e., the associated complex line bundle $G \times \times_{G_{\nu}} \mathbb{C}$. A holomorphic section $s$ of $E$ is a holomorphic map $s: \mathcal{O}_{\nu} \rightarrow E$ with $\pi \circ s(\xi)=\xi$ for all $\xi \in \mathcal{O}_{\nu}$ (here $\pi: E \rightarrow \mathcal{O}_{\nu}$ is the natural projection). Equivalently, $s$ can be viewed as a map $\tilde{s}: G \rightarrow \mathbb{C}$ for which $\tilde{s}(g h)=\chi_{\nu}(h)^{-1} \tilde{s}(g)$ for all $g \in G$ and $h \in G_{\nu}$. For each $\nu \in \mathbf{t}_{+}^{*}$, the set of holomorphic sections $\Gamma(E)$ of the line bundle $E$ over $\mathcal{O}_{\nu}$ is finite. The action of $G$ on $\Gamma(E)$ is given by

$$
g \cdot \tilde{s}(p)=\tilde{s}\left(g^{-1} p\right) \quad \text { for all } g \in G, s \in \Gamma(E) .
$$

Any other irreducible $\rho_{n \nu}$ of $G$ can of course be constructed in the very same way. Note that the line bundle $E^{(n)}$ over $\mathcal{O}_{n \nu} \cong \mathcal{O}_{\nu}$ can also be viewed as the quotient space of $G \times \mathbb{C}^{\otimes n}$ with respect to the equivalence relation

$$
(g h, z) \sim\left(g, \chi_{\nu}^{(n)}(h) z\right) \quad g \in G, h \in G_{\nu}, z \in \mathbb{C}^{\otimes n} .
$$

Here $\chi_{\nu}^{(n)}$ denotes the character $\chi_{\nu}$ extended to the $n$th tensor product $\mathbb{C}^{\otimes n}$ of $\mathbb{C}$.

2.2. The Hardy space $H^{2}(B)$. For our computations we need to have for each ray of representations one unified function space which is exactly the representation space and on which the representations act as Fourier integral operators. Here we describe the construction of such a ray of representations due to [GS1]. 
We denote the $E^{\star}$ be the dual bundle of $E$, i.e., the quotient space of $G \times \mathbb{C}$ with respect to the equivalence relation

$$
(g h, z) \sim\left(g, \chi_{\nu}^{-1}(h) z\right) \quad g \in G, h \in G_{\nu}, z \in \mathbb{C} .
$$

Its disk bundle $D$ is defined by

$$
D=\left\{(\xi, z)\left|\xi \in \mathcal{O}_{\nu}, z \in E_{\xi}^{\star},\right| z \mid \leq 1\right\}
$$

and its circle bundle $B$ by

$$
B=\left\{(\xi, z)\left|\xi \in \mathcal{O}_{\nu}, z \in E_{\xi}^{\star},\right| z \mid=1\right\} .
$$

$D$ is a compact complex domain whose boundary is $B$. Furthermore, $G$ and the circle $S^{1}$ act on $B$ and their actions commute. The Hardy space of $B, H^{2}(B)$, is the space of Cauchy-Riemann (CR) functions on $B$, i.e., the space of $L^{2}$-functions on $B$ that can be extended to holomorphic functions on the whole disk bundle $D$. Holomorphic sections of $E$ correspond to $S^{1}$-equivariant CR functions on $B$ in a one-to-one way: Let $s \in \Gamma\left(E^{\star}\right)$ with $s(\xi)=[g, z], \xi \sim g G_{\nu}$. We then define the corresponding $\hat{s} \in H^{2}(B)$ by

$$
\hat{s}\left(\left[g, e^{i \phi}\right]\right)=z e^{i \phi}, \quad \text { for all } g \in G, e^{i \phi} \in S^{1} .
$$

It is easy to check that $\hat{s}$ is in fact well-defined on $B \sim G \times{ }_{G_{\nu}} S^{1}$ and that $\hat{s}\left(e^{i \theta} \cdot b\right)=$ $e^{i \theta} \hat{s}(b)$ for all $e^{i \theta} \in S^{1}$ and $b \in B$. We denote the set of CR functions $\hat{s}$ on $B$ that can be derived from holomorphic sections in this way by $H_{1}^{2}(B)=\Gamma(E)$. Since $G$ acts on $B$ and this action commutes with the action of $S^{1}, G$ leaves $H_{1}^{2}(B)$ invariant. In fact, $H_{1}^{2}(B)$ is a representation space of the irreducible representation $\rho_{\nu}$ of $G$. By redefining the action of $S^{1}$ on $B$ successively for each $n \in \mathbb{N}$ as

$$
e^{i \theta} \cdot\left[g, e^{i \phi}\right]=\left[g, e^{i n \theta} e^{i \phi}\right],
$$

we arrive at the spaces $H_{n}^{2}(B)=\left\{f \in H^{2}(B) \mid f\left(e^{i \theta} \cdot b\right)=e^{i n \theta} f(b)\right.$ for all $b \in$ $\left.B, e^{i n \theta} \in S^{1}\right\}$ which are each representation spaces of the irreducibles $\rho_{n \nu}$. Since

$$
H^{2}(B)=\bigoplus_{n=1}^{\infty} H_{n}^{2}(B)
$$

this Hardy space is exactly a representation space for the ray through $\rho_{\nu}$.

2.3. The Szegö projector and Fourier-Toeplitz operators. The Szegö projector, i.e. the orthogonal projection

$$
\Pi: L^{2}(B) \rightarrow H^{2}(B)
$$

is a special kind of FIO (of complex type) called a Toeplitz operator. Roughly speaking, a Toeplitz operator is analogous to a pseudodifferential operator, but is microsupported on a symplectic sub-cone $Y \subset T^{*} M$ rather than on the full cotangent bundle.

More generally, we will be considering Toeplitz-like operators which 'quantize' symplectic transformations (and correspondences) on $Y$. Their wave front sets will lie along Lagrangean submanifolds of $Y \times Y$. The basic example is the compression $\Pi \rho(g) \Pi$ of a representation; it corresponds to the classical operation of restricting the group action to the subcone $Y$. Such a Toeplitz operator is the composition of $\Pi$, a standard Toepltiz operator, with the FIO $\rho(g)$. Compositions of Toeplitz operators and FIO's are discussed in detail in BdMG but are not usually included 
under the term 'Toeplitz operator'. We therefore refer to them as 'Fourier-Toeplitz operators'.

The relevant subcone in the case of $\Pi$ is the cone through the prequantum contact structure of $B$. Here, a 1 -form $\alpha$ on a $(2 n-1)$-dimensional manifold $B$ is called a contact form if $\alpha \wedge(d \alpha)^{n-1}$ is a volume form. The halfline subbundle generated by $\alpha$,

$$
Y=\left\{\left(x, r \alpha_{x}\right) \mid x \in B, r \in \mathbb{R}^{+}\right\} \subset T^{*} B,
$$

is then symplectic as a submanifold of $T^{*} B$. In the case of our circle bundle $B$, $\alpha$ is the connection 1-form with curvature $\omega_{\nu}$ for the $S^{1}$-bundle $\pi: B \rightarrow \mathcal{O}_{\nu}$ (see GS2]). It is the unique 1-form invariant under the $S^{1}$ action for which $\iota(\partial / \partial \theta) \alpha=$ 1 and $\iota(\partial / \partial \theta) d \alpha=0$ (here, $\partial / \partial \theta$ denotes the infinitesimal generator of the $S^{1}$ action on $B$ ) The cone $Y$ may also be described as the subspace of $T^{*} B$ given by $\{(x, \xi):\langle v, \xi\rangle=0\}$ for all holomorphic tangent vectors $v \in T_{x} B$ (see [GS1, p. 354].

We then have

Proposition 2.3.1 ([GS1], Proposition 6.5). For all $\nu \in I^{*} \cap \mathbf{t}_{+}^{*}$, the orthogonal projection $\Pi: L^{2}(B) \rightarrow H^{2}(B)$, called the Szegö projector, is a Toeplitz operator (Fourier integral operator of complex type) associated to the identity canonical relation in $Y \times Y$.

The symbol of $\Pi$,

$$
\sigma_{\Pi}=|d y|^{\frac{1}{2}} \otimes \pi_{Y},
$$

is a 1/2-density along $Y$ times a "symplectic spinor" $\pi_{Y}$ in the normal direction $Y^{\perp}$. In fact, $\pi_{Y}$ is a certain rank one projection onto a ground state (Gaussian) associated to $Y^{\perp}$. In the case of discrete random walks, the asymptotics of the spectral measures are determined by traces which kill the symplectic spinor part. The basic reason is that in all the principal terms, the canonical relations will fix $\pi_{Y}$ and then the trace will remove it (since $\operatorname{Tr} \pi_{Y}=1$ ). Hence in the case of discrete random walks it is not necessary to discuss symbols of Toeplitz operators in detail, and we will concentrate only on the $1 / 2$-density $|d y|^{\frac{1}{2}}$. In the case of continuous Lagrangean random walks, the spinor factor is not killed in the trace and plays an important role (see $[\mathrm{PZ}]$ ).

In the proof of our Main Theorem we will need to consider two compositions of FIO's and Toeplitz operators; namely, $T_{g e^{i \theta}} \circ \Pi$ and $\operatorname{Tr} \circ T_{g e^{i \theta}} \circ \Pi$. According to [BdMG, Theorem 9.4], under a clean composition hypothesis both compositions produce Fourier-Toeplitz operators associated to the composite isotropic relations. The general theorem is as follows:

Theorem 2.3.2. Let $K \in I^{r}(X \times Z, \Lambda)$ be a Toeplitz operator or an FIO and $T \in I^{s}(Z \times Y, \Sigma)$ be a Toeplitz operator. We assume that

(i) the composition $\Lambda^{\prime} \circ \Sigma$ is clean (cf. Definition 1.2.1);

(ii) the natural projection $\pi: F \rightarrow \Lambda^{\prime} \circ \Sigma$ has constant rank on each connected component ( $F$ denotes the fiber product of $\Lambda^{\prime}$ and $\Sigma$ ).

Then the composition is a Fourier-Toeplitz operator associated to the isotropic relation $\Lambda^{\prime} \circ \Sigma$, that is

$$
K \circ T \in I^{r+s+\frac{1}{2} e}\left(X \times Z, \Lambda^{\prime} \circ \Sigma\right) .
$$

Example 15. The order of the Szegö projector $\Pi$. Theorem 2.3.2 allows us to compute the order of $\Pi$ : The composition $\Pi \circ \Pi$ is easily seen to be clean. Its 
excess is $e=\operatorname{dim} B-1$. From this and the fact that $\Pi^{2}=\Pi$ we get that $\Pi \in$ $I^{-\frac{1}{2}(\operatorname{dim} B-1)}\left(B \times B, \Delta(Y)^{\prime}\right)$.

Example 16. The composition $T_{e^{i \theta} g} \circ \Pi$. Recall that $T_{e^{i \theta} g} \in I^{-\frac{1}{4}}\left(S^{1} \times B \times\right.$ $B, \Lambda)$ with $\Lambda \cong S^{1} \times T^{*} B$ (see (1.4.6-1.4.7)) and $\Pi$ is the Szegö projector $\Pi \in$ $I^{-\frac{1}{2}(\operatorname{dim} B-1)}\left(B \times B, \Delta(Y)^{\prime}\right)$. We thus have the fiber diagram

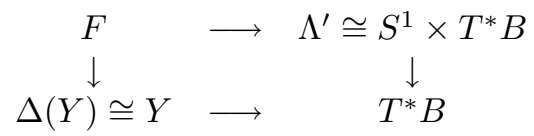

with fiber product

$$
\begin{aligned}
F & =\left\{\left(e^{i \theta},-r ; b, r \alpha ; e^{i \theta} g \cdot b, r \alpha ; e^{i \theta} g \cdot b, r \alpha ; e^{i \theta} g \cdot b, r \alpha\right) \mid r \in \mathbb{R}^{+}, b \in B, e^{i \theta} \in S^{1}\right\} \\
& \cong S^{1} \times Y .
\end{aligned}
$$

Since $d K_{\theta} d L_{g}(v) \in T Y \Leftrightarrow v \in T Y$, the following diagram

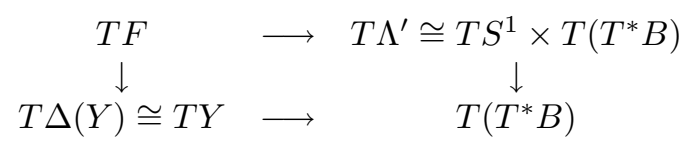

is a fiber diagram with excess $e=0$. We have

$\Lambda_{Y}:=\Lambda^{\prime} \circ \Delta(Y)^{\prime}=\left\{\left(e^{i \theta},-r ; b, r \alpha ; e^{i \theta} g \cdot b, r \alpha\right) \mid r \in \mathbb{R}^{+}, b \in B, e^{i \theta} \in S^{1}\right\} \cong S^{1} \times Y$, and it is obvious that $\pi: F \rightarrow \Lambda_{Y}$ is of constant rank. By Theorem 2.3.2,

$$
T_{e^{i \theta} g} \circ \Pi \in I^{\frac{1}{4}-\frac{1}{2} \operatorname{dim} B}\left(S^{1} \times B \times B, \Lambda_{Y}\right) .
$$

We will also need to discuss the composition principal symbols of FIO's and Toeplitz operators. To avoid unnecessary technicalities, we will only state the composition law for symbols in the special cases that arise in the proof of our Main Theorem.

As in the case of symbol composition for FIO's the symbols of our operators are best described using parametrizations of our Lagrangeans and isotropic relations. As above, the canonical relation $\Lambda$ underlying the FIO $T_{g e^{i \theta}} \in I^{-\frac{1}{4}}\left(S^{1} \times B \times B, \Lambda\right)$ can be parametrized by $S^{1} \times T^{*} B$. Also, the isotropic cone underlying $\Pi$ is the graph of the identity on $Y, \operatorname{Graph}\left(\mathrm{id} 1_{Y}\right)$. Hence the composite Fourier-Toeplitz operator has underlying isotropic relation parametrized by the map

$$
j_{Y}: S^{1} \times Y \rightarrow T^{*} S^{1} \times T^{*} B \times T^{*} B \quad\left(e^{i \theta}, y\right) \rightarrow\left(e^{i \theta}, \Phi(y) ; y ; L_{g} K_{\theta} y\right) .
$$

We recall that $\sigma_{T_{g e} i \theta}=|d \theta|^{\frac{1}{2}} \otimes|\Omega|^{\frac{1}{2}}$ and that $\sigma_{\Pi}=|d y|^{\frac{1}{2}} \otimes \pi_{Y}$. We have

Proposition 2.3.3. With the above notation and assumptions: If the maps $L_{g} K_{\theta}$ preserve $Y$ and if $\left(d L_{g} d K_{\theta}\right)^{*}\left(\sigma_{\Pi}\right)=\sigma_{\Pi}$, then

$$
\sigma_{T_{g i} i \theta} \circ \sigma_{\Pi}=|d \theta|^{\frac{1}{2}} \otimes|d y|^{\frac{1}{2}} \otimes \pi_{Y}
$$

as a symplectic-spinor-valued 1/2-density on $S^{1} \times Y$.

Proposition 2.3.4. With the above notation and assumptions: If $\operatorname{Tr} \circ\left(\Pi \circ T_{g e^{i \theta}}\right)$ is a clean composition of the FIO $\operatorname{Tr}$ and the Fourier-Toeplitz operator $\Pi \circ T_{g e^{i \theta}}$, then the composite isotropic relation is a union $\bigcup_{j=1}^{m} T_{\theta_{j}}^{+*}\left(S^{1}\right)$ of cotangent rays, and the principal symbol of the composition is given on the component $T_{\theta_{j}}^{+*}\left(S^{1}\right)$ by 


$$
\sigma_{\operatorname{Tr} \circ\left(\Pi \circ T_{g e^{i \theta}}\right)}=\operatorname{vol}(B) \xi^{r}|d \xi|^{\frac{1}{2}}
$$

where $r=\frac{1}{2}(e-\operatorname{dim} B)$.

\section{Proof of Main Theorem}

We will determine the asymptotics of the measures by calculating the moments.

The $p$ th moment $M_{r \rho}^{\mu, p}=\int x^{p} d m_{r \rho}^{\mu}$ of the spectral measures $m_{r \rho}^{\mu}, r \in \mathbb{N}$, defined in $(0.4)$ can be written as

$$
M_{r \rho}^{\mu, p}=\left(\operatorname{dim} V_{r \rho}\right)^{-1} \operatorname{Tr} T_{\mu}^{p} 1_{H_{r}^{2}(B)}=\left(\operatorname{dim} V_{r \rho}\right)^{-1} \operatorname{Tr} T_{\mu^{\star p}} 1_{H_{r}^{2}(B)} .
$$

To determine their asympotics, we form the generating functions

$$
\mathcal{Y}_{p}(\theta)=\sum_{r=1}^{\infty} e^{i r \theta} \operatorname{Tr} T_{\mu^{\star p}} 1_{H_{r}^{2}(B)}, \quad p \in \mathbb{N}
$$

which equal

$$
\mathcal{Y}_{p}(\theta)=\operatorname{Tr}\left(T_{e^{i \theta}} \circ T_{\mu^{\star p}} \circ \Pi\right)
$$

with $T_{e^{i \theta}}$ the translation by $e^{i \theta}$ in $L^{2}(B)$. This follows since the differential operator $\frac{1}{i} \frac{\partial}{\partial \theta}$ is equal to $r I$ on $H_{r}^{2}(B)$. The main part of the proof of our theorem will be to show that the $\mathcal{Y}_{p}(\theta)$ are Lagrangean distributions on the circle $S^{1}$. Recalling that Lagrangean distributions on $S^{1}$ have a very specific type of Fourier expansion (see Example 9), this will allow us to determine the asymptotics of the moments. This method of proof is borrowed from $[\overline{\mathrm{BdMG}}$ ] where it was used to prove a Szegö type limit theorem for (pseudodifferential) Toeplitz operators; what we are doing is to generalize the proof to Fourier-Toeplitz operators which arise in random walks (see also TU]).

Recall that $\mu$ is a discrete measure on $G$, hence $\mu^{\star p}$ is discrete for all $p \in \mathbb{N}$. For fixed $p$, we can write $\mu^{\star p}=\sum_{g \in \operatorname{supp} \mu^{\star p}} \mu^{\star p}(g) \delta_{g}$. Hence

$$
T_{\mu^{\star p}}=\sum_{g \in \operatorname{supp} \mu^{\star p}} \mu^{\star p}(g) T_{g} .
$$

In order to show that $\mathcal{Y}_{p}(\theta)$ is a Lagrangean distribution, it therefore suffices to show this for the case $\mu^{\star p}=\delta_{g}$ for some $g \in G$.

We will now verify that the clean composition hypotheses are satisfied in (3.1a) in each composition. The first step is the composition $T_{e^{i \theta}} \circ T_{g}$. The cleanness of this composition has already been proved in Section 1.4. Recall from (1.4.7) that

$$
T_{e^{i \theta}} \circ T_{g} \in I^{-\frac{1}{4}}\left(S^{1} \times B \times B, \Lambda\right) .
$$

Next, we have the compostion of $T_{e^{i \theta}} \circ T_{g}$ with the Szegö projector

$$
\Pi \in I^{-\frac{1}{2}(\operatorname{dim} B-1)}\left(B \times B, \Delta(Y)^{\prime}\right) .
$$

By the discussion in Example 16, it is clean with excess 0 and we have

$$
T_{e^{i \theta}} \circ T_{g} \circ \Pi \in I^{\frac{1}{4}-\frac{1}{2} \operatorname{dim} B}\left(S^{1} \times B \times B, \Lambda_{Y}\right) .
$$

Third, we take the trace of the Fourier-Toeplitz operator $T_{e^{i \theta}} \circ T_{g} \circ \Pi$. As explained in Section 1.5, the operation of taking the trace is itself a composition of Fourier integrals and hence the trace will be a Lagrangean distribution if the composition is clean. We now show that cleanliness of the trace is equivalent to 
cleanliness of the fixed point sets of $T_{g}$ on $\mathcal{O}_{\nu}$ in the sense of Bott (see below for the definition).

To prove this, we first recall (Lemma 1.5.1) that taking the trace corresponds to the compositon of $\Lambda_{Y}^{\prime}$ with the diagonal $\Delta\left(T^{*} B\right)$. To apply Theorem 2.3.2, we must determine when the fiber diagram

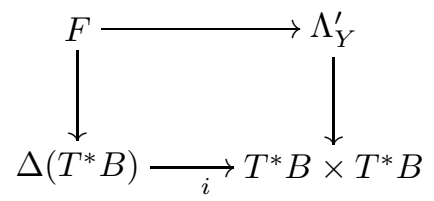

is clean and when $\pi: F \rightarrow \Lambda_{Y}^{\prime} \circ \Delta\left(T^{*} B\right)$ is a map of constant rank on the connected components of $F$.

The fiber product $F$ in the above diagram is clearly

$$
\begin{gathered}
F=\left\{\left(e^{i \phi},-r ; b, r \alpha ; b, r \alpha ; b, r \alpha ; b, r \alpha\right) \mid e^{i \phi} g \cdot b=b, r \in \mathbb{R}\right\} \\
\cong\left\{(b, r \alpha) \mid \exists e^{i \phi} \text { s.t. } e^{i \phi} g \cdot b=b, r \in \mathbb{R}\right\} .
\end{gathered}
$$

Recall that $B$ is an $S^{1}$ bundle over the coadjoint orbit $\mathcal{O}_{\nu}$ and that $G$ and $S^{1}$ act on $B$ via

$$
e^{i \phi} g \cdot b=e^{i \phi} g \cdot\left[h, e^{i \theta}\right]=\left[g h, e^{i \phi+\theta}\right], \quad e^{i \phi} \in S^{1}, g \in G, b=\left[h, e^{i \theta}\right] \in B .
$$

From this we see that

$$
(b, r \alpha) \in F \Leftrightarrow \underline{l}_{g}(\pi(b))=\pi(b)
$$

where $\underline{l}_{g}$ denotes the action of $g$ on $\mathcal{O}_{\nu}$ and $\pi$ is the natural projection $\pi: B \rightarrow \mathcal{O}_{\nu}$. Let us denote the fixed point set of $\underline{l}_{g}$ by $\operatorname{Fix}\left(\underline{l}_{g}\right)$. Thus we have

$$
F \cong\left\{(b, r \alpha) \mid b \in \pi^{-1}\left(\operatorname{Fix}\left(\underline{l}_{g}\right)\right), r \in \mathbb{R}\right\} ;
$$

hence $F$ is a manifold if $\operatorname{Fix}\left(\underline{l}_{g}\right)$ is a manifold. We also need to determine when the diagram

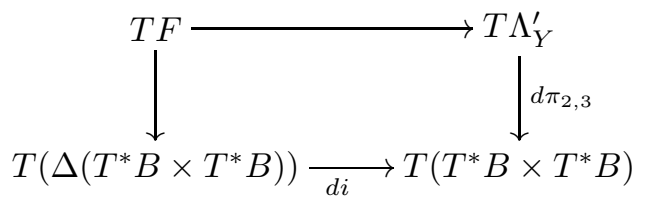

is a fiber diagram. Now, for any $v \in T \Lambda_{Y}^{\prime} \cong T\left(S^{1} \times Y\right)$,

$$
d \pi_{2,3}(v) \in T\left(\Delta\left(T^{*} B \times T^{*} B\right)\right) \Leftrightarrow d l_{e^{i \phi}} d l_{g}(w)=w,
$$

$w$ being the orthogonal projection of $v$ onto $T B$. The contact form $\alpha$ is a connection one form on $T B$, i.e., at each $b \in B$, the kernel of $\alpha$ defines a horizontal subspace Hor $_{b} \subset T_{b} B$ with Hor $_{b} \cong T_{\pi(b)} \mathcal{O}_{\nu}$. Furthermore, $T_{b} B=$ Hor $_{b} \oplus \mathrm{V}_{b}$ where $\mathrm{V}_{b}$ is the one-dimensional tangent space along the fiber $S^{1}$,

$$
\begin{aligned}
& d l_{g}\left(\operatorname{Hor}_{b}\right)=\operatorname{Hor}_{g \cdot b}, \quad d l_{g}\left(\mathrm{~V}_{b}\right)=\mathrm{V}_{g \cdot b} \quad \text { and } \\
& d l_{e^{i \phi}}\left(\operatorname{Hor}_{b}\right)=\operatorname{Hor}_{e^{i \phi} \cdot b}, \quad d l_{e^{i \phi}}\left(\mathrm{V}_{b}\right)=\mathrm{V}_{e^{i \phi} \cdot b} .
\end{aligned}
$$

From all this it follows that (3.7) is a clean fiber diagram if

1) $\operatorname{Fix}\left(\underline{l}_{g}\right)$ is a manifold and

2) for all $x G_{\nu} \in \operatorname{Fix}\left(\underline{l}_{g}\right), T_{x G_{\nu}}\left(\operatorname{Fix}\left(\underline{l}_{g}\right)\right)=\operatorname{Fix}\left(d \underline{l}_{g}\right)_{x G_{\nu}}$.

This says precisely that $l_{g}$ has clean fixed point manifolds. 
The next step is to show that for all $g \in G, \underline{l}_{g}$ has clean fixed point manifolds on $\mathcal{O}_{\nu}$. Note that it suffices to show this for all $g \in T$, where $T$ is the chosen maximal torus. Indeed, each element $h \in G$ is conjugate to an element $g \in T$, i.e., $h=k g k^{-1}$ for some $k \in G$. This implies that $\operatorname{Fix}\left(\underline{l}_{h}\right)=k \cdot \operatorname{Fix}\left(\underline{l}_{g}\right)$ and condition 2 holds for $\underline{l}_{g}$ if and only if it holds for $\underline{l}_{h}$.

At times we will distinguish two types of elements in $T$, regular elements and singular elements. An element $g$ is called regular, if its powers generate T. Almost all (with respect to Haar measure) elements in $T$ are regular. On the other hand, there is the set of singular elements defined as the union of the kernels of the positive roots (see [BtD], Section V.2). A singular element $g$ generates a closed subgroup of $T$ of dimension strictly smaller than $\operatorname{dim} T$.

The case $\nu \in$ interior $I^{*} \cap \mathbf{t}_{+}^{*}$. In case $\nu \in$ interior $I^{*} \cap \mathbf{t}_{+}^{*}$, the interior of the positive Weyl chamber, we have $G_{\nu}=T$ and thus $\mathcal{O}_{\nu} \cong G / T$. For all $y T \in \mathcal{O}_{\nu}$ we have

$$
g y T=y T \quad \Leftrightarrow \quad y^{-1} g y \in T \quad \Leftrightarrow \quad y=z w \text { with } w \in W_{G} \text { and } z \in Z(g)
$$

where $W_{G}$ denotes the Weyl group of $G$ and $Z(g)$ denotes the centralizer of $g$. The centralizer $Z(g)$ is always a closed subgroup of $G$. Note that

$$
g \text { regular } \Leftrightarrow Z(g)=T .
$$

This shows that the fixed point set is given by $\operatorname{Fix}\left(\underline{l}_{g}\right)=\left\{z w T \mid z \in Z(g), w \in W_{G}\right\}$, hence is a closed submanifold of $\mathcal{O}_{\nu}$. (In case $g$ is regular, $\operatorname{Fix}\left(\underline{l}_{g}\right)=\{w T \mid z \in$ $\left.Z(g), w \in W_{G}\right\} \cong W_{G}$ is a discrete set and independent of $g$.) This proves condition 1.

Let us now prove condition 2. First, we show that condition 2 holds at $e T \in$ $\operatorname{Fix}\left(\underline{l}_{g}\right)$. The tangent space $T_{e T} \mathcal{O}_{\nu}$ is isomorphic to the subspace $L(G / T):=$ $\bigoplus_{\beta \in R^{+}} M_{\beta}$ of the Lie algebra $g$. Here $R^{+}$denotes the set of positive roots and $M_{\beta}$ denotes the 2-dimensional rootspace corresponding to the $\operatorname{root} \beta$. Furthermore, the Lie algebra $L(Z(g))$ of the centralizer $Z(g)$ is the direct sum

$$
L(Z(g))=\mathbf{t} \oplus \bigoplus_{\beta \in N} M_{\beta}
$$

where $\mathbf{t}$ denotes the Lie algebra of $T$ and $N$ denotes the set of positive roots $\beta$ whose kernel contains $g$ ( $N$ is empty for $g$ regular). See, for example, BtD, Proposition V.2.3. The tangent space of $\operatorname{Fix}\left(\underline{l}_{g}\right)$ at $e T$ is therefore isomorphic to $\bigoplus_{\beta \in N} M_{\beta}$. Under the identification of $T_{e T} \mathcal{O}_{\nu}$ with $L(G / T)=\bigoplus_{\beta \in R^{+}} M_{\beta}$, the differential $d \underline{l}_{g}: T_{e T} \mathcal{O}_{\nu} \rightarrow T_{e T} \mathcal{O}_{\nu}$ is the adjoint representation $\operatorname{Ad}(g)$ at $g$, restricted to $\bigoplus_{\beta \in R^{+}} M_{\beta}$. But by definition of $N$, the subspace consisiting of fixed points of $\operatorname{Ad}(g)$ is exactly the space $\bigoplus_{\beta \in N} M_{\beta}$. This proves that $\operatorname{Fix}\left(d \underline{l}_{g}\right)_{e T}=T_{e T} \operatorname{Fix}\left(\underline{l}_{g}\right)$.

We now show that condition 2 holds at any other point in $\operatorname{Fix}\left(\underline{l}_{g}\right)$. Observe that for any $z w T \in \operatorname{Fix}\left(\underline{l}_{g}\right)$,

$$
T_{z w T} \mathcal{O}_{\nu} \cong T_{e / T} \mathcal{O}_{\nu} \quad \text { via } \quad v \in T_{e T} \mathcal{O}_{\nu} \leftrightarrow d \underline{l}_{z} d \underline{r}_{w}(v) \in T_{z w T} \mathcal{O}_{\nu}
$$

where $\underline{r}_{w}$ denotes the map $\underline{r}_{w}: \mathcal{O}_{\nu} \rightarrow \mathcal{O}_{\nu}, \underline{r}_{w}(x T)=x w T$. Since $\underline{r}_{w}$ and $\underline{l}_{z}$ commute with $\underline{l}_{g}$,

$$
v \in \operatorname{Fix}\left(d \underline{l}_{g}\right)_{e T} \quad \Leftrightarrow \quad d \underline{l}_{z} d \underline{r}_{w}(v) \in \operatorname{Fix}\left(d \underline{l}_{g}\right)_{z w T}
$$

and in particular 


$$
\operatorname{dim}\left(\operatorname{Fix}\left(d \underline{l}_{g}\right)_{e T}\right)=\operatorname{dim}\left(\operatorname{Fix}\left(d \underline{l}_{g}\right)_{z w T}\right)
$$

which proves 2) for all $g$.

The case $\nu \notin$ interior $I^{*} \cap \mathbf{t}_{+}^{*}$. In this case $\nu$ lies in at least one wall of the fundamental Weyl chamber, i.e., there exist $\alpha \in R^{+}$, the set of positive roots, such that $\alpha(\mu)=0$. (We have identified $\mathbf{t}^{*}$ with $\mathbf{t}$ via an inner product on $\mathbf{t}$.) The Lie algebra $L\left(G_{\nu}\right)$ of the stabilizer group $G_{\nu}$ is

$$
L\left(G_{\nu}\right)=\mathbf{t} \oplus \bigoplus_{\beta \in K} M_{\beta}
$$

where $K:=\left\{\alpha \in R^{+} \mid \alpha(\mu)=0\right\}$. This follows from the fact that $G_{\mu}=Z(s)$ for all singular elements $s=\exp X \in T$ for which $\alpha(X)=0$ for all $\alpha \in K$ and $\alpha(X) \neq 0$ for all $\alpha \in K^{c}:=R^{+} \backslash K$. We therefore have

$$
T_{e G_{\nu}} \mathcal{O}_{\nu} \cong \bigoplus_{\beta \in K^{c}} M_{\beta}
$$

For any $g \in T$ and any $y G_{\nu} \in \mathcal{O}_{\nu}$ we claim that the following holds:

$$
\begin{aligned}
g y G_{\nu}=y G_{\nu} & \Leftrightarrow y^{-1} g y \in G_{\nu} \\
& \Leftrightarrow y=z w u \text { with } z \in Z(g), w \in W G, \text { and } u \in G_{\nu} .
\end{aligned}
$$

Indeed, $y^{-1} g y \in G_{\nu}$ implies that there exists a $u \in G_{\nu}$ such that $u y^{-1} g y u^{-1} \in T$ (since $T$ is also a maximal torus of $G_{\nu}$ ). Furthermore, there exists a $w \in W_{G}$ such that $w u y^{-1} g y u^{-1} w^{-1}=g$. But this implies that $w u y^{-1} \in Z(g)$, from which the claim follows.

So far we have shown that the fixed point set is $\operatorname{Fix}\left(\underline{l}_{g}\right)=\left\{z w G_{\nu} \mid z \in Z(g)\right.$, $w \in W G\}$ and hence is a closed submanifold of $\mathcal{O}_{\nu}$. In order to prove that condition 2 holds, we can apply the same ideas as in the previous case. Again, we consider the decomposition of $L(Z(g))$ as in (3.9): $L(Z(g))=\mathbf{t} \oplus \bigoplus_{\beta \in N} M_{\beta}$. This provides us with the isomorphism

$$
T_{e G_{\nu}} \operatorname{Fix}\left(\underline{l}_{g}\right) \cong \bigoplus_{\beta \in N \backslash K} M_{\beta}
$$

Under this isomorphism, the differential $d_{\underline{l}}: T_{e G_{\nu}} \mathcal{O}_{\nu} \rightarrow T_{e G_{\nu}} \mathcal{O}_{\nu}$ is the adjoint representation $\operatorname{Ad}(g)$ at $g$, restricted to $\bigoplus_{\beta \in K^{c}} M_{\beta}$. But the fixed point set of Adg restricted to $T_{e G_{\nu}}$ is exactly $\bigoplus_{\beta \in N \backslash K} M_{\beta}$. This proves that $\operatorname{Fix}\left(d \underline{l}_{g}\right)_{e G_{\nu}}=$ $T_{e G_{\nu}} \operatorname{Fix}\left(\underline{l}_{g}\right)$. The rest is completely analogous to the case $\nu \in$ interior $I^{*} \cap \mathbf{t}_{+}^{*}$.

The rank of the map $\pi: F \rightarrow \Lambda_{Y}^{\prime} \circ \Delta\left(T^{*} B\right)$ is constant and equal to 1 . This follows easily from the fact that the action of $S^{1}$ and the action of $G$ on $Y$ are both homogeneous in $\mathbb{R}$ (recall (3.4)).

We have now proved that the trace operation is clean and thus that $\mathcal{Y}_{p}(\theta)$ is a Lagrangean distribution on $S^{1}$. We still need to compute the excess of this composition. From (3.3) and the definition of the excess $e$ we have

$$
e=\operatorname{dim} F+\operatorname{dim}\left(T^{*} B \times T^{*} B\right)-\left(\operatorname{dim} \Delta\left(T^{*} B\right)-\operatorname{dim} \Lambda_{Y}^{\prime}\right) .
$$

We know that a Lagrangean distribution on the circle $S^{1}$ has only a finite number of singular points $\theta_{j}, j=1, \ldots, K$. Among those singular $\theta_{j}$ 's, the ones for which 
$e^{i \theta_{j}} g$ fixes the whole circle bundle $B$ produce the connected components of highest possible dimension, namely $\operatorname{dim} Y$, of $F$. For $b=\left[h, e^{i \phi}\right] \in B$ we have

$$
\begin{gathered}
e^{i \theta} g \cdot\left[h, e^{i \phi}\right]=\left[g h, e^{i(\theta+\phi)}\right]=\left[h, e^{i \phi}\right] \quad \Leftrightarrow \quad \exists t \in G_{\nu} \\
\text { such that } g h=h t \text { and } e^{i \theta}=\chi_{\nu}(t) .
\end{gathered}
$$

Thus, if the fixed point set $\operatorname{Fix}\left(e^{i \theta} g\right)=B$ it follows that

$$
g \in Z_{\nu}:=\bigcap_{h \in G} h G_{\nu} h^{-1} .
$$

Hence we have,

$$
\operatorname{Fix}\left(e^{i \theta} g\right)=B \quad \text { iff } \quad \nu \in Z_{\nu} \quad \text { and } \quad e^{i \theta}=\chi_{\nu}(g) .
$$

Moreover, $Z_{\nu}=Z_{G}$ if $\nu$ is an interior point, since $G_{\nu}$ is then a maximal torus and the intersection of all maximal torii is the center. Provided that $\operatorname{Fix}\left(e^{i \theta} g\right)=B$, for at least one $\left(e^{i \theta}, g\right)$, the excess of the trace operation is

$$
e=\operatorname{dim} Y+4 \operatorname{dim} B-2 \operatorname{dim} B-(1+\operatorname{dim} Y)=2 \operatorname{dim} B-1 .
$$

From this, (3.2), and the fact that the trace operation $\operatorname{Tr} \in I^{0}\left(S^{1} \times B \times B \times\right.$ $\left.S^{1}, \Delta\left(T^{*} S^{1} \times T^{*} B\right)\right)$ we get

$$
\mathcal{Y}_{p}(\theta) \in I^{\frac{1}{2} \operatorname{dim} B-\frac{1}{4}}\left(S^{1}, \bigcup_{j=1}^{K} T_{\theta_{j}}^{+*} S^{1}\right) .
$$

Remark. We should note that often $Z_{\nu}=Z_{G}$ even when $\nu$ is a wall weight. For instance, in the case of $G=U(n)$ this happens as long as $\nu$ is not a scalar weight (i.e. a weight of the form $(r, r, \ldots, r))$. Indeed, $g \in \bigcap_{h \in G} h G_{\nu} h^{-1}$ implies that for all $h \in G, g$ commutes with $h \nu h^{-1}$ and thus with any $h \pi h^{-1}$ where $\pi$ denotes any spectral projection of $\nu$. Here, we are regarding elements of $G$, of $\mathbf{g}$, thus also of $\mathbf{t}^{*}$, as endomorphisms of $\mathbb{C}^{n}$. If $\nu$ is not a scalar, there exists an eigenvalue $a$ of $\nu$ whose eigenspace $K$ (the range the spectral projection $\pi_{a}$ ) has dimension $k$ with $k<n$. Since for all $h \in G, g$ commutes with $h \pi_{a} h^{-1}, g$ leaves each $k$ dimensional subspace $h(K), h \in G$, invariant. But this implies that $g$ leaves every $k$ dimensional subspace invariant ( $G$ acts transitively on the Grassmann manifolds). We claim this is only possible if $g$ is a scalar, i.e. if $g \in Z_{G}$. Indeed suppose $g$ is not a scalar. Then there is at least one vector $x \in \mathbb{R}^{n}\left(\mathbb{C}^{n}, \mathbb{H}^{\mathrm{n}}\right)$ for which $g(x)=c x+y$ with $y \perp x, y \neq 0$. We then can find a $k$ dimensional subspace $W$ containing $x$ but not containing $y$, which is a contradiction to $g(W) \subset W$.

The situation for the other classical groups $S O(n), S p(n)$ is more or less analogous (with almost complex structures $J$ playing the role of scalar weights).

Recall now Example 9. Since $\mathcal{Y}_{p}(\theta)$ is polyhomogeneous and a Hardy distribution (the wave front set is contained in a union of positive half spaces), it can be written as a sum

$$
\mathcal{Y}_{p}(\theta)=\sum_{j=0}^{\infty} \sum_{l=1}^{K} a_{j, l} \chi_{m-j}\left(\theta-\theta_{l}\right)
$$

of the basic homogeneous distributions $\chi_{r}$ discussed in Example 8. Provided that $a_{0, l} \neq 0$ for at least one $l$, this means that $\mathcal{Y}_{p}(\theta) \in I^{m+\frac{1}{4}}\left(S^{1}, \bigcup_{l=1}^{K} T_{\theta_{l}}^{+*} S^{1}\right)$. We 
easily find that $m=\frac{1}{2}(\operatorname{dim} B-1)$ with the use of (3.17). From (3.18) we get

$$
\operatorname{Tr} T_{\mu^{\star p}} 1_{H_{r}^{2}(B)}=r^{m} \sum_{l=1}^{K} a_{0, l} e^{-i r \theta_{l}}+r^{m-1} \sum_{l=1}^{K} a_{1, l} e^{-i r \theta_{l}}+\text { lower order terms . }
$$

On the other hand, by Proposition 2.3.4 of Section 2.3,

$$
\begin{aligned}
& \sigma\left(\mathcal{Y}_{p}\right)=\sum_{\left(i_{1}, \ldots, i_{p}\right)} c_{i_{1}} \ldots c_{i_{p}} \sigma_{\operatorname{Tr} \Pi T_{g_{i_{1}} \ldots g_{i_{p}} e^{i \theta}}} \\
= & \sum_{\left(i_{1}, \ldots, i_{p}\right)} \operatorname{vol}(B) c_{i_{1}} \ldots c_{i_{p}} \sigma\left(\chi_{m}\right)\left(\theta_{g_{i_{1}} \ldots g_{i_{p}}}\right) .
\end{aligned}
$$

Since a Lagrangean distribution of order $m$ with vanishing principal symbol actually has order $m-1$, we conclude that

$$
\mathcal{Y}_{p}=\sum_{\substack{\left(i_{1}, \ldots, i_{p}\right): \\ g_{i_{1}} \ldots g_{i_{p}} \in Z_{\rho}}} \operatorname{vol}(B) c_{i_{1}} \ldots c_{i_{p}} \chi_{m}\left(\theta-\theta_{g_{i_{1}} \ldots g_{i_{p}}}\right)+\text { smoother }
$$

and hence by $(3.18)$ we have $a_{0, l}=c_{i_{1}} \ldots c_{i_{p}} \operatorname{vol}(B)$. By comparing Fourier series, we find that

$$
\operatorname{Tr} T_{\mu^{\star p}} 1_{H_{r}^{2}(B)} \sim C \operatorname{vol}(B) r^{m} \sum_{\substack{\left(i_{1}, \ldots, i_{p}\right): \\ g_{i_{1}} \ldots g_{i_{p}} \in Z_{\rho}}} c_{i_{1}} \ldots c_{i_{p}} e^{-i r \theta_{g_{i_{1}} \ldots g_{i_{p}}}}+O\left(r^{m-1}\right)
$$

with $e^{-i r \theta_{g_{i_{1}} \ldots g_{i_{p}}}}=\chi_{\rho}\left(g_{i_{1}} \ldots g_{i_{p}}\right)$ and with $C$ a universal constant. After normalizing by taking the quotient with $\operatorname{dim} H_{r}^{2}(B)=\operatorname{dim} V_{r \rho}$ the coefficient $r^{m} C \operatorname{vol}(B)$ cancels and we get

$$
\frac{\operatorname{Tr} T_{\mu^{\star p}} 1_{H_{r}^{2}(B)}}{\operatorname{dim} H_{r}^{2}(B)}=\sum_{\substack{\left(i_{1}, \ldots, i_{p}\right): \\ g_{i_{1}} \ldots g_{i_{p}} \in Z_{\rho}}} c_{i_{1}} \ldots c_{i_{p}} e^{-i r \theta_{g_{i_{1}} \ldots g_{i_{p}}}}+o(1)
$$

completing the proof of the Main Theorem.

The proofs of Corollaries 1 and 2 were given in the introduction. The proof of Corollary 3 is essentially the combination of Corollary 1 with known results on the spectrum of $\lambda\left(T_{\mu}\right)$ (the spectrum of the induced random walk on $l^{2}(\Gamma)$ ). For the sake of completeness, we include the proof:

Proof of Corollary 3. (i) Kesten's amenability criterion for countable groups $\Gamma$ states that

$$
\Gamma \text { is an amenable group } \quad \Leftrightarrow \quad \sup \operatorname{supp} \gamma=1
$$

where $\gamma$ is the spectral measure of the random walk $(\Gamma, \mu)$ (see $[\mathrm{Ke}$, $\underline{\mathrm{KV}}$, Section 5.1]). Suppose that $\beta_{\rho}^{*}<1, \rho \neq$ scalar, and $\chi_{\rho}(z) \neq 1$ for $z \in Z_{G} \cap \Gamma \backslash\{i d\}$. Consider an arbitrary continuous function $f$ with support in the interval $\left[\beta_{\rho}^{*}, 1\right]$. It follows that

$$
\int f d m_{r \rho}^{\mu}=0 \quad \forall r \in \mathbb{N} .
$$

On the other hand, by (0.5),

$$
\int f d m_{r \rho}^{\mu}=\sum_{z \in Z_{G} \cap \Gamma} \chi_{\rho}(z)^{r} \int f d m^{\mu, z}+O\left(r^{-1}\right) .
$$


But $\int f d m^{\mu, e}>0\left(m^{\mu, e}\right.$ is Kesten's spectral measure for $\left.(\Gamma, \mu)\right)$, so, by our assumptions on the $\chi_{\rho}(z)$, the right hand side in (3.19) is unequal to zero, which is a contradiction.

(ii) Let $\Gamma$ be a free countable group and $\mu$ a symmetric probability measure on $\Gamma$ supported on the set of generators $\left\{a_{i}^{ \pm 1}, i=1, \ldots, n\right\}$ with $\left\{a_{i}, i=1, \ldots, n\right\}$ free. A theorem by Kesten ([Ke, Theorem 3]) states that

$$
\sigma(\Gamma, \mu)=\left[-\frac{\sqrt{2 n-1}}{n}, \frac{\sqrt{2 n-1}}{n}\right] .
$$

Suppose now that $\left[-\frac{\sqrt{2 n-1}}{n}, \frac{\sqrt{2 n-1}}{n}\right] \not \subset \overline{\sigma\left(T_{\mu} 1_{\mathbb{N} \rho}\right)}$. It follows that there exists an interval $[c, d] \subset\left[-\frac{\sqrt{2 n-1}}{n}, \frac{\sqrt{2 n-1}}{n}\right]$ with $[c, d] \cap \overline{\sigma\left(T_{\mu} 1_{\mathbb{N} \rho}\right)}=\emptyset$. Consider an arbitrary continuous function $f$ with support in $[c, d]$. The same argument as in (i) shows that integrating both sides in (0.5) against $f$ leads to a contradiction.

\section{EXAMPLES}

4.1. Random walk on the torus $T^{k}=\left(S^{1}\right)^{k}$. Let $G=T^{k}$ and

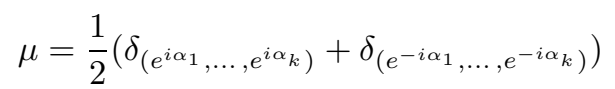

with $\left(\alpha_{1}, \ldots, \alpha_{k}\right) \notin(2 \pi \mathbb{Q})^{k}$. All irreducible representations of $T^{k}$ are one dimensional. They correspond to the lattice points $(\mathbb{Z})^{k} \subset \mathbb{R}^{k}$. For a given $\rho=$ $\left(n_{1}, \ldots, n_{k}\right)$, the eigenvalues of $T_{\mu}$ along the ray $\mathbb{N} \rho$ can easily be computed as $\lambda_{r \rho}=\cos \left(r\left(n_{1} \alpha_{1}+\ldots+n_{k} \alpha_{k}\right)\right)(r \in \mathbb{N})$. By the Main Theorem, we have

$$
d m_{r \rho}^{\mu}=\delta_{\cos \left(r\left(n_{1} \alpha_{1}+\ldots+n_{k} \alpha_{k}\right)\right)} \sim \sum_{z \in T^{k}} \chi_{\rho}(z)^{r} d m_{\infty \rho}^{\mu, z}+O\left(r^{-1}\right)
$$

with $\chi_{\rho}(z)^{r}=e^{r i\left(n_{1} z_{1}+\ldots+n_{k} z_{k}\right)}$ for $z=\left(e^{i z_{1}}, \ldots, e^{i z_{k}}\right)$. The $d m_{\infty \rho}^{\mu, z}$ are determined by the moments

$$
\int_{\mathbb{R}} x^{p} d m_{\infty \rho}^{\mu, z}=\mu^{* p}(\{z\})
$$

and are zero unless $z=\left(e^{i l \alpha_{1}}, \ldots, e^{i l \alpha_{k}}\right)$ for some $l \in \mathbb{Z}$. Note that for fixed $z=\left(e^{i l \alpha_{1}}, \ldots, e^{i l \alpha_{k}}\right)$,

$$
\mu^{* p}(z)= \begin{cases}\frac{\left(\begin{array}{c}
p \\
p-|l|
\end{array}\right)}{2}\left(\frac{1}{2}\right)^{p} & \text { for }|l| \leq p \text { and }(p-|l|) \text { even } \\
0 & \text { otherwise }\end{cases}
$$

No weak limit exists for $m_{r \rho}^{\mu}$ as $r \rightarrow \infty$. Since $T^{k}$ is an abelian group, $\Gamma$ is amenable, and by Corollary $2(\mathrm{i})$, the spectral radius $\beta_{\rho}^{*}=1$. (This can, of course, also be seen directly from the fact that $\left\{e^{r\left(n_{1} \alpha_{1}+\ldots+n_{k} \alpha_{k}\right)} \mid r \in \mathbb{N}\right\}$ is dense in $S^{1}$.)

4.2. Random walk on the sphere. The special orthogonal group $S O(n+1)$ acts transitively on the $n$-dimensional unit sphere $S^{n} \cong S O(n+1) / S O(n)$ in $\mathbb{R}^{n+1}$. Through this action, we get a representation of $S O(n+1)$ on the Hilbert space $L^{2}\left(S^{n}\right)$ which is exactly a ray, namely

$$
L^{2}\left(S^{n}\right)=\mathbb{N} \rho_{1}
$$

where $\rho_{1}$ is the $(n+1)$-dimensional natural representation. This follows since $L^{2}\left(S^{n}\right) \cong L^{2}(S O(n+1) / S O(n))$ is an induced representation, namely the trivial 
representation $\tilde{\rho_{0}}$ of $S O(n)$ induced up to $S O(n+1)$. By Frobenius reciprocity,

$$
\text { multiplicity of } \rho \in L^{2}\left(S^{n}\right)=\text { multiplicity of } \tilde{\rho_{0}} \in \operatorname{res}_{S O(n)}^{S O(n+1)} \rho \text {. }
$$

By the familiar branching rules for the restiction of an irreducible of $S O(n+1)$ to $S O(n)$ (see, for example, [FH $\S 25.3]$ ), we have

$$
\text { multiplicity of } \tilde{\rho_{0}} \in \operatorname{res}_{S O(n)}^{S O(n+1)} \rho \leq 1
$$

and

$$
\tilde{\rho_{0}} \in \operatorname{res}_{S O(n)}^{S O(n+1)} \rho \Leftrightarrow \rho=r \rho_{1}, r \in \mathbb{N} .
$$

(Although $L^{2}\left(S^{n}\right)$ is a ray of representations, it is not presented as the Hardy space of a prequantum circle bundle $B$ over an orbit $\mathcal{O}$. Nevertheless, the methods of this paper apply to it, and in fact in a more elementary form; there is no need to introduce a Toeplitz projector. Otherwise, all of the previous calculations remain valid.)

4.3. A few rank 2 groups. (a) $G=U(2)$. The maximal torus of the unitary group $U(2)$ is the subgroup of diagonal matrices $T=\left\{\operatorname{diag}\left(e^{i \phi_{1}}, e^{i \phi_{2}}\right) \mid \phi_{1}, \phi_{2} \in\right.$ $[0,2 \pi)\} \cong U(1) \times U(1)$ and its center is the subgroup of scalar matrices $Z_{U(2)}=$ $\left\{\operatorname{diag}\left(e^{i \phi}, e^{i \phi}\right) \mid \phi \in[0,2 \pi)\right\} \cong U(1)$. There is only one positive root, $\left(e_{2}-e_{1}\right)$ $\left(e_{i}\right.$ denotes the $i$ th standard basis vector of $\mathbb{R}^{2}$. The positive Weyl chamber is $\mathbf{t}_{+}^{*}=\left\{\left(x_{1}, x_{2}\right): x_{2} \geq x_{1}\right\} \subset \mathbb{R}^{2}$; it has a unique wall $x_{2}=x_{1}$. Below is a picture of $\mathbf{t}_{+}^{*}$ and a few rays.

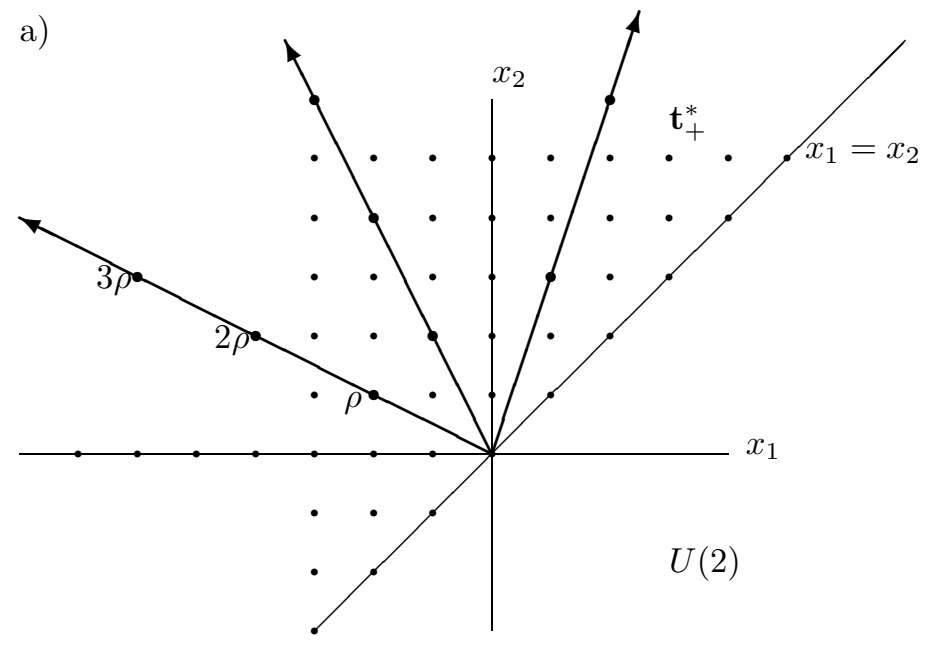

The orbit corresponding to an interior lattice point $\nu$ is $\mathcal{O}_{\nu}=U(2) / T=U(2) / U(1)$ $\times U(1)=S U(2) / U(1)$ which is the 2 -sphere $S^{2}$ (equivalently, complex projective space $\left.\mathbb{C} P^{1}\right)$. The prequantum $S^{1}$ bundle $B$ is then $S U(2)$ itself and the projection to $S^{2}$ is the usual Hopf projection from $S^{3} \rightarrow S^{2}$.

For a lattice point $\psi$ in the wall, we have $G_{\psi}=U(2)$. Thus the orbit $\mathcal{O}_{\psi}$ is just one point. It follows that $B=S^{1}$, so the Hardy space is just the classical Hardy space of the unit circle, i.e., $H^{2}(B)=\left\{\sum_{r=1}^{\infty} a_{r} e^{i r \theta} \mid \sum_{r=1}^{\infty} a_{r}^{2}<\infty\right\}$. 
(b) $G=S O(4)$. The maximal torus is the subgroup of block diagonal matrices

$$
T=\left\{\operatorname{diag}\left(\left(\begin{array}{cc}
\cos \phi_{1} & -\sin \phi_{1} \\
\sin \phi_{1} & \cos \phi_{1}
\end{array}\right) \times\left(\begin{array}{cc}
\cos \phi_{2} & -\sin \phi_{2} \\
\sin \phi_{2} & \cos \phi_{2}
\end{array}\right)\right)\right\} \cong S O(2) \times S O(2)
$$

and the center is $Z_{S O(4)}=\{ \pm I\} \cong Z_{2}\left(Z_{S O(n)} \cong Z_{2}\right.$ for any even $\left.n\right)$. A positive root system is $R^{+}=\left\{\left(e_{2}-e_{1}\right),\left(e_{2}+e_{1}\right)\right\}$; this gives rise to the positive Weyl chamber $\mathbf{t}_{+}^{*}=\left\{\left(x_{1}, x_{2}\right): x_{2} \geq\left|x_{1}\right|\right\}$. Picture $\left.\mathrm{b}\right)$ below shows $\mathbf{t}_{+}^{*}$ and a few rays. The lattice point corresponding to the natural representation $\rho_{1}$ is $(0,1)$ and lies in the interior. The coadjoint orbit $\mathcal{O}_{\nu}$ for any interior $\nu$ is the Grassmann manifold $G_{2,4}(\mathbb{R})=S O(4) / S O(2) \times S O(2)$.

(c) $G=S O(5)$. The maximal torus is the subgroup of block diagonal matrices $T=\left\{\operatorname{diag}\left(1 \times\left(\begin{array}{cc}\cos \phi_{1} & -\sin \phi_{1} \\ \sin \phi_{1} & \cos \phi_{1}\end{array}\right) \times\left(\begin{array}{cc}\cos \phi_{2} & -\sin \phi_{2} \\ \sin \phi_{2} & \cos \phi_{2}\end{array}\right)\right)\right\} \cong S O(2) \times S O(2)$

and the center is trivial $\left(Z_{S O(n)}\right.$ is always trivial for $n$ odd). A positive root system is $R^{+}=\left\{\left(e_{2}-e_{1}\right),\left(e_{2}+e_{1}\right), e_{2}, e_{1}\right\}$; this gives rise to the positive Weyl chamber $\mathbf{t}_{+}^{*}=\left\{\left(x_{1}, x_{2}\right): x_{2} \geq x_{1} \geq 0\right\}$ (see picture c) below). Here the lattice point $(0,1)$ corresponding to the natural representation $\rho_{1}$ lies in the wall $x_{1}=0$. The stabilizer group $G_{\rho_{1}}$ is $S O(3) \times S O(2)$. It follows that the coadjoint orbit $\mathcal{O}_{\rho_{1}}$ is the Grassmann manifold $G_{3,5}(\mathbb{R})=S O(5) / S O(3) \times S O(2)$.

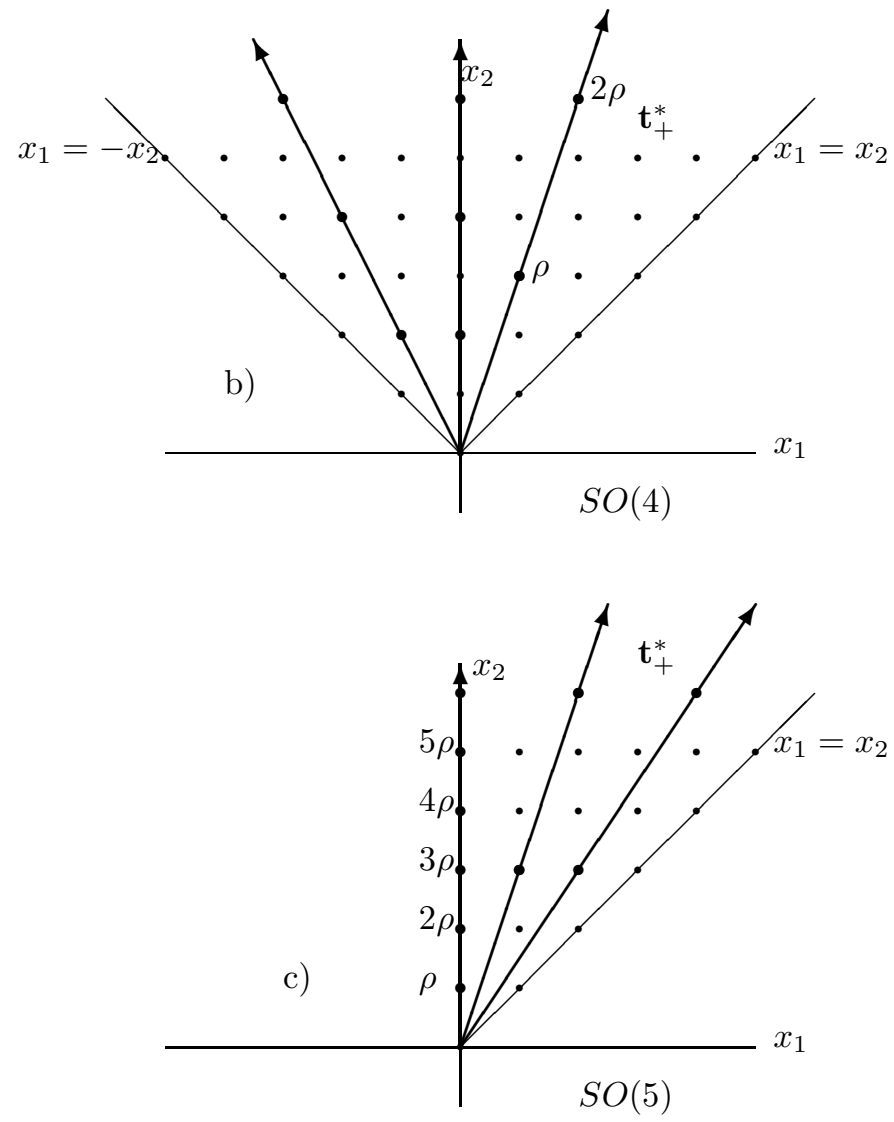


4.4. Pseudo-Harper operators. As mentioned in the introduction, an interesting example of a random walk on the unitary group $U(q)$ is provided by the image of a rational Harper operator $H_{\theta}\left(\theta=\frac{p}{q}\right)$ under the distinguished irreducible unitary representation of the rotation algebra $A_{\theta}$ taking $U$ to $u=\operatorname{diag}\left(1, e^{i \theta}, \ldots, e^{i(q-1) \theta}\right)$ and $V$ to the cyclic permutation $v \in U(q)$ of the standard (eigenvector) basis $e_{1}, \ldots, e_{q}$. We recall that the rotation algebra $A_{\theta}$ is the algebra generated by unitaries $U, V$ satisfying $U V=e^{2 \pi i \theta} V U$. The elements $u, v$ of $U(q)$ determine the probability measure

$$
\mu=\frac{1}{4}\left(\delta_{u}+\delta_{u^{*}}+\delta_{v}+\delta_{v^{*}}\right)
$$

on $U(q)$ and hence the transition operator $H^{2}\left(B_{\rho}\right)$ given by

$$
T_{\mu}=\frac{1}{4}\left(T_{u}+T_{u^{*}}+T_{v}+T_{v^{*}}\right)
$$

with $T_{g}$ the translation operator by $g$ on $H^{2}\left(B_{\rho}\right)$.

We recall from the introduction that

$$
T_{u} T_{v}=T_{z_{o}} T_{v} T_{u}
$$

where $z_{o}=e^{2 \pi i \theta}$ is in the center of $U(q)$. Since this is not the commutation relation of $A_{\theta}$ we cannot expect $T_{\mu}$ to have a subspectrum of $H_{\theta}$. Nevertheless, the spectrum of $T_{\mu}$ can be determined along any ray of representations of $U(q)$ from the spectral theory of Harper operators. In the following $S p$ denotes the spectrum in the usual sense as a closed set.

Proposition 4.4.1. With the notations above: Along any ray of representations $\mathbb{N} \rho, S p\left(T_{\mu} 1_{H^{2}\left(B_{\rho}\right)}\right)=[-1,1]$ as a set. In fact, it is the union of the spectra of $q$ Harper operators, including the commutative Harper operator.

Proof. We begin by relating the commutation relation (4.4.1) along subrays to those of certain rotation algebras. Observe, then, that at the $r$ th representation, say $V_{r \rho}$, along the ray $\mathbb{N} \rho$ the center $Z_{U(q)}=\{z I: z \in U(1)\}$ acts by the scalar $\chi_{\rho}(z)^{r}$. Indeed, if we let $f(g, w)$ denote a function on $G \times U(1)$ which is invariant under the action of $G_{\rho}$ defining $B_{\rho}:=(G \times U(1)) / G_{\rho}$, and equivariant of degree $r$ under the action of $U(1)$ on $B_{\rho}$, then

$$
T_{z} f(g, w)=f(z g, w)=f\left(g, \chi_{\rho}(z) w\right)=\chi_{\rho}^{r}(z) f(g, w) .
$$

Since $V_{r \rho}$ consists of the degree $r$ CR-functions on $B_{\rho}$ (under the $U(1)$-action), we see that on $V_{r \rho}$ the commutation relations for $T_{u}, T_{v}$ read:

$$
T_{u} T_{v}=\chi_{\rho}\left(z_{o}\right)^{r} T_{v} T_{u} \quad z_{o}=e^{2 \pi i \theta} .
$$

Here $\chi_{\rho}\left(z_{o}\right)^{r}$ denotes multiplication by this scalar. We note that if $\rho=\left(\lambda_{1}, \ldots, \lambda_{q}\right)$, then $\chi_{\rho}\left(e^{2 \pi i \theta}\right)=e^{2 \pi i \theta \bar{\rho}}$ with $\bar{\rho}=\lambda_{1}+\cdots+\lambda_{q}$. Thus, $T_{u}, T_{v}$ generate the rotation algebra $A_{r \theta \bar{\rho}}$ on $V_{r \rho}$.

We further observe that when $\theta=\frac{p}{q}$, the multiplier $e^{2 \pi i r \theta \bar{\rho}}$ is periodic of period $q$ in $r$. Of course it might be periodic of a smaller period if $\bar{\rho}$ is not relatively prime to $q$. However we always have that

$$
H^{2}\left(B_{\rho}\right)=\bigoplus_{m=0}^{q-1} H_{m}^{2}
$$


with

$$
H_{m}^{2}:=\bigoplus_{r \equiv m \bmod q} V_{r \rho}
$$

and with $u, v$ satisfying the commutation relation

$$
u v=e^{2 \pi i m \theta \bar{\rho}} v u
$$

on $H_{m}^{2}$.

Let us henceforth assume for the sake of simplicity that $(\bar{\rho} p, q)=1$. We will call such a ray a "relatively prime ray" for $T_{\mu}$. Also, let $T_{\mu, m}$ denote the restriction of the random walk to the 'sub-ray' $H_{m}^{2}$. The main step in the proof of the proposition is contained in the following:

Lemma 4.4.2. $S p T_{\mu, m}=S p H_{\frac{p^{\prime}}{q^{\prime}}}$ where $\frac{p^{\prime}}{q^{\prime}}=\frac{m p \bar{\rho}}{q}$.

Proof. We first recall that $S p H_{\frac{p^{\prime}}{q^{\prime}}}$, the spectrum of $H_{\frac{p^{\prime}}{q^{\prime}}}$ as an element of $A_{\frac{p^{\prime}}{q^{\prime}}}$, is the union of the spectra of the $q^{\prime} \times q^{\prime}$ matrices $\pi_{z_{1}, z_{2}}\left(H_{\frac{p^{\prime}}{q^{\prime}}}\right)$ which arise in the irreducible representations of $A_{\frac{p^{\prime}}{q^{\prime}}}$. We recall that for $\theta=\frac{p^{\prime}}{q^{\prime}},\left(p^{\prime}, q^{\prime}\right)=1$, the irreducible representations of $A_{\theta}$ are all of dimension $q^{\prime}$ and are parametrized, with some redundancies, by the 2-torus $T^{2}$ (see [CEY p. 227]). One irreducible representation $\pi$ is defined by $\pi(U)=\operatorname{diag}\left(1, \eta, \eta^{2}, \ldots, \eta^{q-1}\right)$ where $\eta=e^{2 \pi i \theta}$ and by putting $\pi(V)$ equal to the cyclic permutation operator on the eigenvector basis $e_{1}, \ldots, e_{q}$. The other representations $\pi_{z_{1}, z_{2}}$ are obtained by composing $\pi$ with the automorphism $\alpha_{z_{1}, z_{2}}$ defined by $U \rightarrow z_{1} U, V \rightarrow z_{2} V$. Thus, the spectrum of $H_{\frac{p^{\prime}}{q^{\prime}}}$ is simply a union of spectra of finite matrices.

On the other hand, the spectrum of $T_{\mu, m}$ is also a union of spectra of finite matrices, namely the matrices $T_{\mu} 1_{V_{r \rho}}$ occurring along the points of the subray. These matrices are the images of $T_{\mu}$ in the irreducible representations of $A_{\frac{p^{\prime}}{q^{\prime}}}$ occurring in the subray. Hence $S p\left(T_{\mu, m}\right)$ is the union of the spectra of these matrices, taken over all the irreducibles occurring along the ray. In particular, $S p\left(T_{\mu, m}\right) \subset S p\left(H_{\frac{p^{\prime}}{q^{\prime}}}\right)$.

To prove equality, it would suffice to show that the set of irreducibles of $A_{\frac{p^{\prime}}{q^{\prime}}}$ occurring along the subray is dense in the set $T^{2}$ of all irreducibles. This is not implausible, since the dimensions $d_{r}=\operatorname{dim} V_{r \rho}$ of the irreducibles of $U(q)$ along the ray are growing at a polynomial rate, while all the irreducibles of $A_{\frac{p^{\prime}}{q^{\prime}}}$ are of dimension $q^{\prime}$. Hence, $V_{(m+n q) \rho}$ are highly reducible as representations of $A_{\frac{p^{\prime}}{q^{\prime}}}$, at least for $n>>1$. The set of irreducibles of $A_{\frac{p^{\prime}}{q^{\prime}}}$ occurring in $H_{m}^{2}$ thus determines a countably infinite subset $\hat{A}_{\frac{p^{\prime}}{q^{\prime}}, \rho, m}$ of the torus $T^{2}$ parametrizing all of the irreducibles, and the closure of the union of all the eigenvalues of $H_{\frac{p^{\prime}}{q^{\prime}}}$ (as we range over $\left.\hat{A}_{\frac{p^{\prime}}{q^{\prime}}, \rho, m}\right)$ gives the spectrum of the random walk along the $m$ th subray.

However, we can argue more directly, using the Main Theorem. To this end, we reconsider the asymptotic formulae for the spectral measures $m_{r \rho}^{\mu}$ as a sum of measures $m^{\mu, z}$ with the oscillatory coefficients $\chi_{\rho}(z)^{r}$, where $z$ ranges over $\Gamma \cap Z_{U(q)}$. We note that $u v u^{*} v^{*}=z_{0}:=e^{2 \pi i \frac{p}{q}}$ lies in $\Gamma \cap Z_{U(q)}$ and that all other intersection points are powers of $z_{0}$. Indeed, to lie in the center, a word in the letters $u, v$ must consist of a string of $u, u^{*}, v, v^{*}$ with an equal number of $u, u^{*}$ 's and $v, v^{*}$ 's. 
Cancelling out we see that all the central elements lie in the cyclic group generated by $z_{0}$. We can therefore rewrite the asymptotic formula for $m_{(m+n q) \rho}^{\mu}$ in the form

$$
m_{(m+n q) \rho}^{\mu} \sim \sum_{a \in \mathbb{N}} \chi_{\rho}\left(z_{0}^{a}\right)^{m} m^{\mu, z_{0}^{a}} \quad(n \rightarrow \infty)
$$

in the sense that

$$
\int_{\mathbb{R}} x^{k} d m_{(m+n q) \rho}^{\mu}=\sum_{a \in \mathbb{N}} e^{2 \pi i m a \bar{\rho} \frac{p}{q}} \mu^{* k}\left(\left\{z_{0}^{a}\right\}\right)+o(1) .
$$

We observe that the principal term on the right hand side is independent of $n$ and hence gives a well-defined (non-oscillatory) asymptote along each of the subrays.

Moreover, the asymptote is identifiable as the spectral measure for the Harper operator in its "magnetic translation representation" [B], [BVZ], [Su]. In this representation $U, V$ get taken to the unitary operators on $l^{2}\left(\mathbb{Z}^{2}\right)$ defined by

$$
(U \phi)(m, n)=e^{-2 \pi i \alpha_{2} m} \phi(m, n+1) \quad(V \phi)(m, n)=e^{-2 \pi i \alpha_{1} n} \phi(m+1, n)
$$

where $\theta=\alpha_{2}-\alpha_{1}$. In the physical interpretation, the pair $\left(\alpha_{1}, \alpha_{2}\right)$ define the vector potential $\alpha_{1} y d x+\alpha_{2} x d y$ of a magnetic field $B=\left(\alpha_{2}-\alpha_{1}\right) d x \wedge d y$ whose flux through a period cell of the lattice is $\theta$. The Harper operator $H_{\theta}=\frac{1}{4}\left(U+U^{*}+V+V^{*}\right)$ has then a spectrum as an operator on $l^{2}\left(\mathbb{Z}^{2}\right)$, and it coincides with the spectrum of this operator as an element of the rotation algebra $A_{\theta}$.

Recall now that there exists a unique trace $\tau$ on $A_{\theta}$, namely

$$
\tau \sum a_{m, n} U^{m} V^{n}=a_{o, o}=\left\langle\sum a_{m, n} U^{m} V^{n} \delta_{0}, \delta_{0}\right\rangle
$$

where 0 denotes the origin of $\mathbb{Z}^{2}$ (cf. $[\mathrm{B}$, $\underline{\mathrm{Su}}]$ ). We can thus define a spectral measure for $H_{\theta}$ via the formula

$$
\mu_{\theta}(f):=\tau\left(f\left(H_{\theta}\right)\right) \quad f \in C\left(S p\left(H_{\theta}\right)\right) .
$$

It is closely related to the spectral measure for the discrete random walk defined by $H_{\theta}$ on the Heisenberg group (cf. [BVZ])

We then observe that $d m_{\theta}$ is essentially the same measure as the asymptote to $d m_{(m+n q) \rho}^{\mu}$ in (4.4.6a) when $\theta=\frac{p^{\prime}}{q^{\prime}}$ as above. To see this, we compare moments. In the case of $d m_{\theta}$ we can compute these by associating to any monomial in $\left(U+V+U^{*}+V^{*}\right)^{k}$ a path in the lattice $\mathbb{Z}^{2}$ starting at the origin and then determined by the following traffic directions: reading from right to left in the monomial, go right at $U$, left at $U^{*}$, up at $V$, and down at $V^{*}$. We then observe that only monomials giving rise to loops (i.e., closed paths starting and ending at the origin) contribute to the moments. Hence only even moments are non-zero. Using $U V=e^{2 \pi i \theta} V U$, we see that the contribution of such "loop-monomials" to the $2 k$ th moment has the form

$$
\sum_{a \in \mathbb{N} \text { loops } \in \Gamma_{2 k}} \chi_{\rho}\left(z_{o}^{a}\right)^{m} \#\left\{2 k \text {-loops }: z_{\text {loop }}=z_{o}^{a}\right\} .
$$

Here, $\Gamma_{2 k}$ is the set of elements of word-length $2 k$ and for a word representing a "loop", $z_{\text {loop }}$ is the element of the center to which the word reduces. We have also used the definition of $\frac{p^{\prime}}{q^{\prime}}$. Comparing to the leading asymptote of $d m_{(m+n q) \rho}^{\mu}$ we see that both spectral measures have precisely the same moments. It follows that they have the same supports. 
Since the spectrum of $H_{\theta}$ equals the support of $m_{\theta}$, we conclude that the spectrum of $T_{\mu, m}$ contains the spectrum of $H_{\theta}$, where as above $\theta=\frac{p^{\prime}}{q^{\prime}}$. Combined with the reverse inclusion noted above, the proof of the lemma is complete.

To complete the proof of the proposition, we note that

$$
S p\left(T_{\mu} 1_{H_{\rho}^{2}(B)}\right)=\bigcup_{m=0}^{q-1} S p\left(T_{\mu} 1_{H_{m, \rho}^{2}(B)}\right) .
$$

In the case of $m=0, T_{\mu} 1_{H_{m, \rho}^{2}(B)}$ is Harper operator corresponding to the commutative Harper algebra, and its spectrum is that of the multiplication operator $\frac{1}{2}(\cos x+\cos y)$, namely $[-1,1]$. For the other values of $m, S p\left(T_{\mu} 1_{H_{m, \rho}^{2}(B)}\right)$ equals the spectrum of the Harper operator $H_{\frac{m p \bar{p}}{q}}$ and hence is the union of bands separated by gaps as described in $\left[\mathrm{B},[\mathrm{CEY}]\right.$. It follows that $S p\left(T_{\mu} 1_{H_{\rho}^{2}(B)}\right)$ is a dense pure point spectrum in $[-1,1]$ whose closure has a well-defined multiplicity function obtained from the comparison with the $q$ Harper operators.

\section{REFERENCES}

[BVZ] C. Beguin, A. Valette, and A. Zuk, On the spectrum of a random walk on the discrete Heisenberg group and the norm of Harper's operator, J. Geom. Phys. 21 (1997), 337-356. MR 99e:60025

[B] J. Bellissard, Noncommutative methods in semiclassical analysis, Springer Lecture Notes in Mathematics 1589, Transition to Chaos (S. Graffi, ed.), (1994). MR 96e:81050

[BtD] T. Bröcker and T. tom Dieck, Representations of Compact Lie Groups; Graduate Texts in Math., Springer-Verlag New York, 1985. MR 86i:22023

[BdMG] L. Boutet de Monvel and V. Guillemin, The Spectral Theory of Toeplitz Operators; Princeton University Press, Princeton, NJ, 1981. MR 85j:58141

[CEY] M-D. Choi, G. A. Elliott, and N. Yui, Gauss polynomials and the rotation algebra, Inventiones Math. 99, 225-246 (1990). MR 91b:46067

[CV] Y. Colin de Verdiere, Distribution de points sur une sphère, Sém. Bourbaki 41 (1988-89) no. 703, in Asterisque 177-78, 83-93 (1989). MR 91k:11089

[D] J. J. Duistermaat, Fourier Integral Operators; Courant Institute of Mathematical Sciences Lecture Notes, New York, 1973. MR 56:9600

[DG] J. J. Duistermaat and V. Guillemin, The spectrum of positive elliptic operators and periodic bicharacteristics; Inventiones Math. 29, 39-79 (1975). MR 53:9307

$[\mathrm{FH}] \quad$ W. Fulton and J. Harris, Representation Theory, A First Course; Graduate Texts in Mathematics 129, Springer-Verlag, New York, 1991. MR 93a:20069

[GSj] A. Grigis and J. Sjöstrand, Microlocal Analysis for Differential Operators; London Math. Society Lecture Note Series 196, Cambridge University Press, 1994. MR 95d:35009

[G] V. Guillemin, Lectures on spectral theory of elliptic operators; Duke Math. J. 44 No. 3, 485-517 (1977). MR 56:6758

[GS1] V. Guillemin and S. Sternberg, Homogeneous quantization and multiplicities of group representations; J. Funct. Anal. 47 No. 3, 344-380 (1982). MR 84d:58034

[GS2] V. Guillemin and S. Sternberg, Geometric quantization and multiplicities of group representations; Inventiones Math. 67 515-538 (1982). [MR 83m:58040]

[GS3] V. Guillemin and S. Sternberg, Geometric Asymptotics; Amer. Math. Soc., Providence, 1977. MR 58:24404

[HRV] P. de la Harpe, A. G. Robertson, and A. Valette, On the spectrum of the sum of generators of a finitely generated group, Israel J.Math. 81 (1993), 65-96. MR 94j:22007

[Ho] L. Hormander, The Analysis of Linear Partial Differential Operators I-IV; Springer-Verlag, Berlin, Heidelberg, 1983-1985. MR 85g:35002a; MR 85g:35002b MR 87d:35002a MR 87d:35002b

$[\mathrm{KV}] \quad$ V. A. Kaimanovich and A. M. Vershik, Random walks on discrete groups: boundary and entropy; Annals Prob. 11 No. 3, 457-490 (1983). MR 85d:60024 
[Ke] H. Kesten, Symmetric random walks on groups; Trans. Amer. Math. Soc. 92, 336-354 (1959). MR 22:253

[Ki] A. A. Kirillov, Elements of the theory of representations; Grundlehren der mathematischen Wissenschaften 220, Springer-Verlag, Berlin, New York, 1976. MR 54:447

[LPS] A. Lubotzky, R. Phillips, and P. Sarnak, Hecke operators and distributing points on the sphere I; Comm. Pure Appl. Math. 39 No. S, suppl., S149-S186 (1986). MR 88m:11025a

[N] K. Nomizu, Lie Groups and Differential Geometry; The Mathematical Society of Japan, Tokyo, 1956. MR 18:821d

[P] U. Porod, The cut-off phenomenon for random reflections, Annals of Prob. 24 (1996), 74-96. MR 97e:60012

[PZ] U. Porod and S. Zelditch, Semiclassical limit of random walks. II, Asymptotic Analysis 18, 215-261 (1998). CMP 99:08

[R] J. Rosenthal, Random rotations: characters and random walks on $S O(N)$; Annals Prob. 22 No. 1, 398-423 (1994). MR 95c:60008

[Stein] E. M. Stein, Harmonic Analysis, Princeton Mathematical Series, 43, Princeton University Press, Princeton, 1993. MR 95c:42002

[Su] T. Sunada, A discrete analogue of periodic magnetic Schrodinger operators, in Geometry of the Spectrum, Amer. Math. Soc. Contemp. Math., 173, (R. Brooks, C. Gordon, P. Perry, eds.) Amer. Math. Soc. (1994). IMR 95i:58185

[TU] M. E. Taylor and A. Uribe, Semiclassical spectra of gauge fields, J. Funct. Anal. 110 (1992), 1-46. MR 95d:58143

[T] F. Treves, Introduction to Pseudodifferential and Fourier Integral Operators. II; Plenum Press, New York, 1980. MR 82i:35173

[Z] J. Zak, Magnetic translation group I, II; Phys. Rev. A 134, 1602-1611 (1964). MR 31:2032

Department of Mathematics, University of California, Berkeley, California 94720

Department of Mathematics, Johns Hopkins University, Baltimore, Maryland 21218 